\title{
Proposta di un catalogo visuale di modelli per lo studio della forma architettonica tra Matematica e Disegno
}

\author{
Caterina Cumino \\ Martino Pavignano \\ Ursula Zich
}

\begin{abstract}
"I 'trattati' [...] devono essere visti come un'integrazione essenziale ai modelli. Prima di tutto, forniscono al docente tutto ciò che è necessario per un loro uso efficace: istruzioni per l'uso e informazioni sul loro impiego in classe e riferimenti bibliografici. Inoltre, vi sono incluse le questioni, alle quali già il più semplice dei modelli offre occasione" [Wiener 1907, p. 2].

Una rilettura critica di cataloghi di superfici matematiche di fine $X I X /$ inizio $X X$ secolo si è rivelata fonte di ispirazione per l'esplorazione delle Geometria come linguaggio condiviso per indagare l'Architettura e per una sistematizzazione di modelli tangibili nati come ausili formativi per la comprensione e rappresentazione della forma architettonica.

La ricerca si inserisce nell'alveo del più articolato progetto di ricerca interdisciplinare MAG.IA, Matematica Architettura Geometria. Interconnessioni Applicative e un esito è la creazione di un apparato grafico descrittivo e progettuale che rende accessibili tali modelli, mediante un catalogo visuale.

L'analisi puntuale di alcuni dei suddetti repertori storici ha evidenziato inoltre l'estrema attualità di tale approccio nel momento in cui la dematerializzazione di molti supporti per la didattica, in diretta correlazione alla necessità di offrire formazione in modalità remota, spinge verso una digitalizzazione massiva a discapito di una pratica manuale e tangibile che è implicita nella produzione di modelli fisici.
\end{abstract}

Parole chiave

catalogo, modelli tangibili, geometria, rappresentazione, forma architettonica.

Esempio di catalogazione di modelli di superfici: Katalog mathematischer und mathematisch-phy1892, p.286] cata [Dick 1892, p. 286], catalogo proposto dagli autori ne contributo.

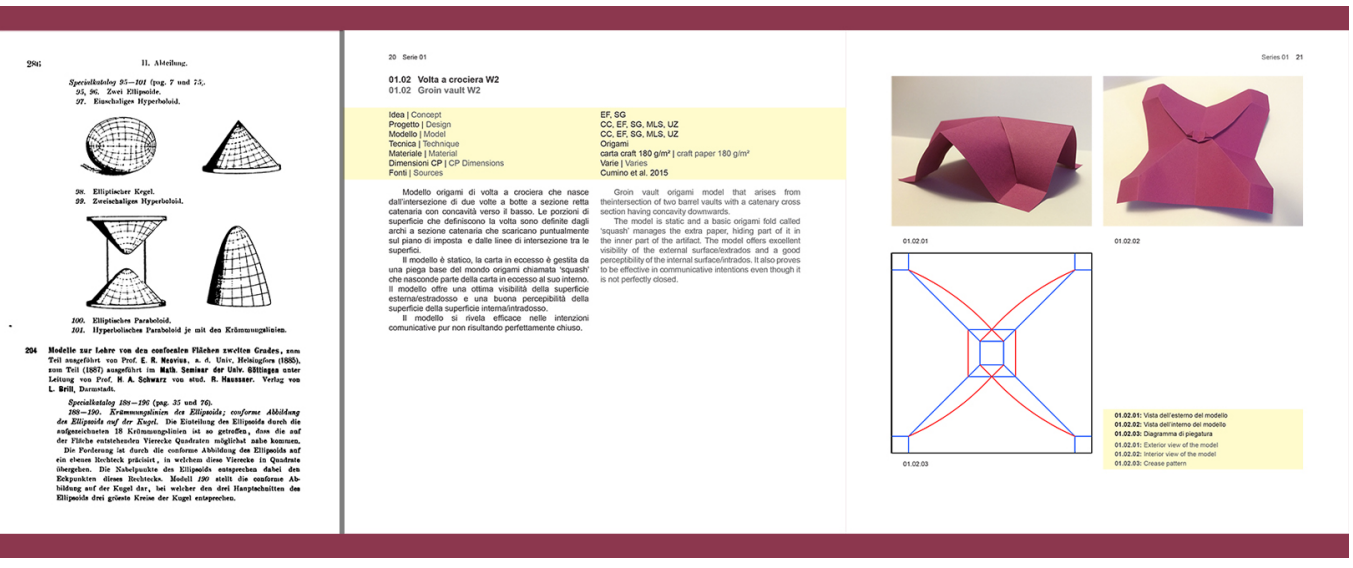




\section{Introduzione}

La Geometria costituisce un ambito comune tra Matematica e Architettura: nella prima la Geometria si occupa di spazi astratti, mentre nella seconda si occupa dello spazio concreto [Quaroni 1978] e "l'Architettura [...] che in ogni sua operazione adopera le misure, dipende dalla Geometria, e vuol sapere almeno i primi suoi elementi”' [Guarini I737, p. 3]. La Geometria, nei secoli, ha sviluppato metodi e modelli sempre più raffınati e articolati, descrivibili per mezzo di vari linguaggi, che corrispondono a diverse specializzazioni: alcune interessano maggiormente l'architetto, da un punto di vista applicativo; per esempio, la Geometria Sintetica, che analizza gli oggetti del piano e dello spazio euclidei dal punto di vista del procedimento sotteso alla loro costruzione, è forse la più congeniale alla forma mentis degli architetti in quanto capace di dialogare con il loro principale linguaggio cioè con il Disegno [De Fusco 2010; Cardone 2016] e, come tale, è alla base della Geometria Descrittiva. Proprio nel contesto universitario della Geometria Descrittiva, si sviluppa in Europa nel XIX secolo un grande interesse per la produzione di ausili tattili e visivi per l'insegnamento pratico della teoria. Fiere mondiali e rispettive mostre -da Londra I 85 I a Saint Louis 1904- erano contesto dove strumenti matematici, modelli e macchine per il calcolo e per il disegno, venivano esposti al pubblico; erano occasioni per diffondere una cultura materiale in una dimensione geopolitica che ruotava intorno agli oggetti matematici, un contesto dove la comunità matematica promuoveva scambi di idee e tecnologia [Giacardi 20 I5] e non solo, dal momento che il primo catalogo di Schilling ( 1903) introduceva alcuni modelli di oggetti architettonici (fig. I) utili allo sviluppo delle capacità di copia dal vero e di riconoscimento delle geometrie costituenti [Wiener 19l।]. Ma i progettisti erano anche venditori e i cataloghi di modelli di superfici matematiche i loro registri per la promozione, divenuti poi memoria del fervore culturale intorno alla produzione dei modelli. Hermann Wiener, professore di Geometria Descrittiva a Karlsruhe, divenne noto per i suoi modelli matematici che presentava alle esposizioni di ausili per la didattica della matematica [Wiener 1907, p. 3]. Oggi quegli oggetti, divenuti patrimonio delle collezioni di istituzioni e musei scientifici, sono spesso poco accessibili, molte volte mediati dalle dinamiche di fruizione on line che ne condizionano l'essenza offrendo una esperienza indiretta di un prodotto tangibile. D'altra parte, al di là della curio-

Fig. I. Esempi di descrizioni visuo-testuali dal Catalog mathematischer Modelle di Martin Schilling (1903): VI Infinitesimalgeometrie der Flachen (p. 103); VIII. Modelle (p. $\mid 14)$.

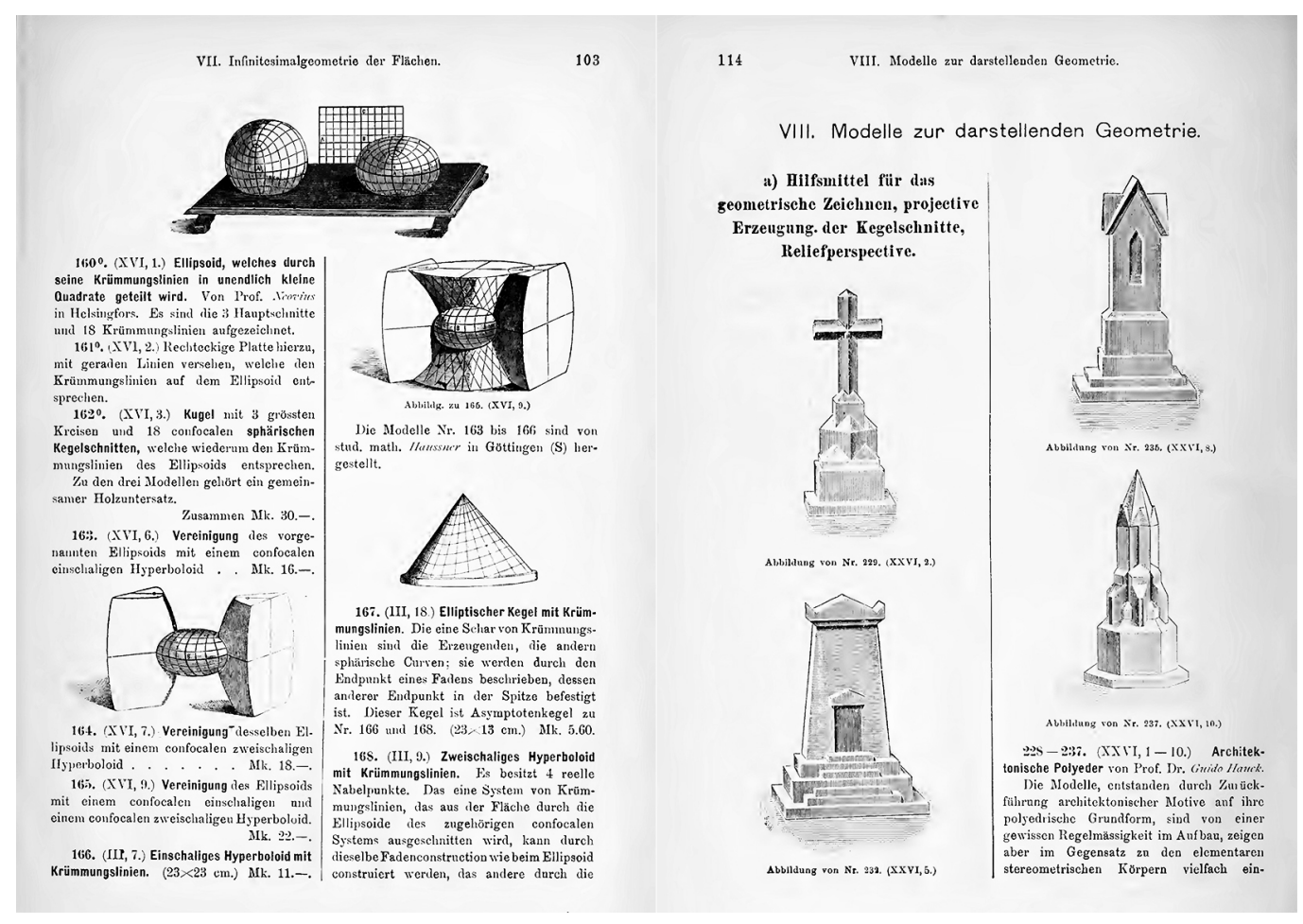


Fig. 2. Rassegna sintetica di copertine rappresentativa dei cataloghi di modeli matematici, dal 1872 a 1961, riletti criticamente. Fabre de Lagrange, Merriefiled (1872), Dick (1892) Schilling (1903) (I892), Schilling (1903), Wiener (1907), Schilling (1911), Wiener (1911), Wiener, Treutlein (1912) (1920-1928), Stoll (1961) sità da wunderkammer che essi possono suscitare nel pubblico contemporaneo, sembrano tuttora meritevoli di attenzione da parte della comunità scientifica, non tanto per le loro prerogative puramente matematiche, quanto piuttosto per la loro caratteristica di artefatti visuali [Gay 2017, p. 95] utili per formulare una sorta di linguaggio visivo della Geometria. In tal senso, nell'esplorare le relazioni tra Matematica e Disegno per identificare le modalità per la comprensione e la rappresentazione delle forme architettoniche, abbiamo sviluppato negli anni metodologie che si appoggiano al modello fisico come esito della mediazione tra i due approcci culturali/scientifici. Una rilettura critica dei cataloghi di superfici matematiche di fine $X \mid X /$ inizio $X X$ secolo è stata per noi fonte di ispirazione per una sistematizzazione degli artefatti fin qui prodotti e per la creazione di un apparato grafico descrittivo e progettuale che rendesse accessibili tali modelli, mediante un catalogo visuale. L'analisi puntuale di questi repertori ha evidenziato l'estrema attualità di tale approccio in un momento in cui la necessità di una didattica in remoto induce la dematerializzazione di molti ausili didattici a discapito della tangibilità implicita nella produzione di modelli fisici (fig. 2).
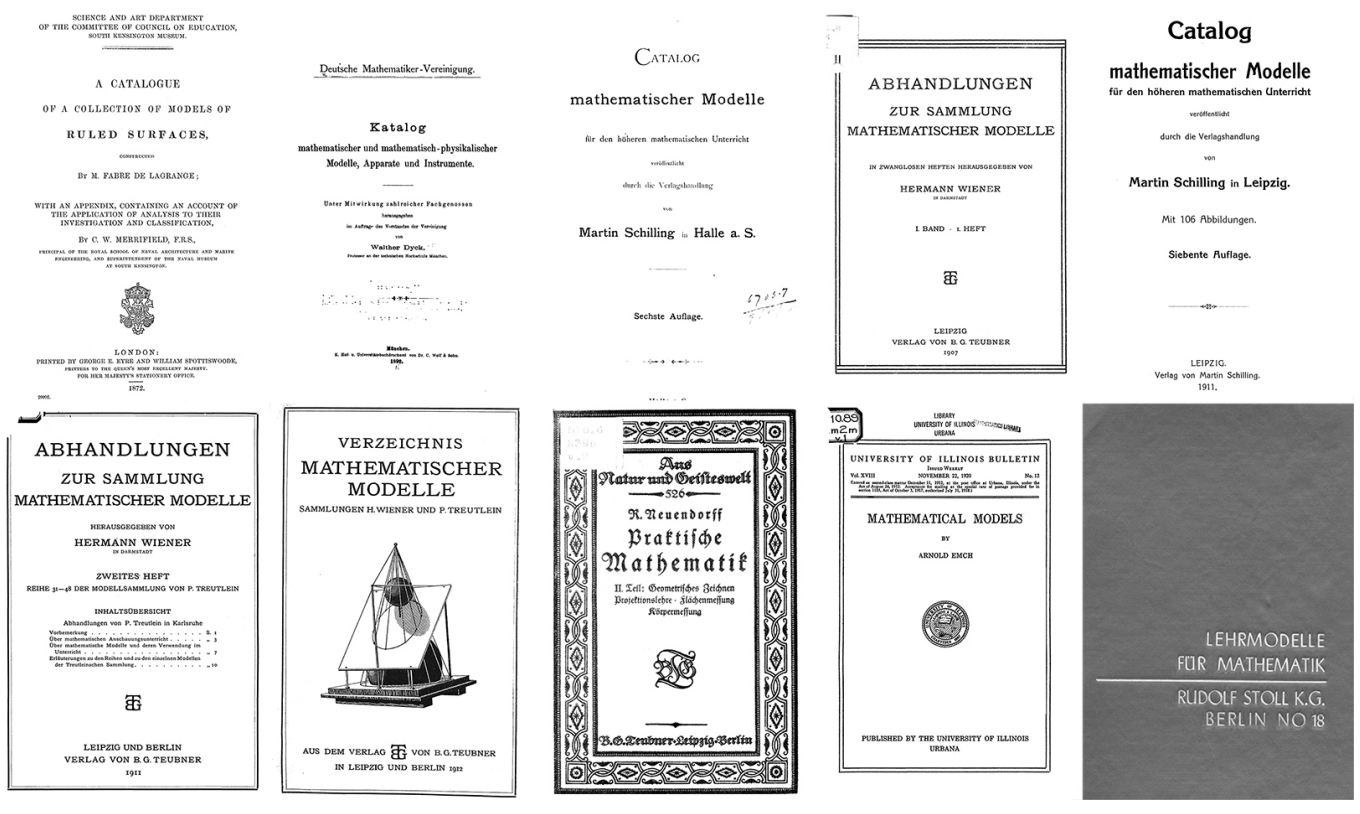

\section{Uno sguardo sulle collezioni scientifiche in rete}

II panorama italiano è ricco di collezioni scientifiche variamente esposte in rete. Tra gli Istituti che esplorano le potenzialità del virtuale, I'Università "la Sapienza" di Roma [I] mette a disposizione numerosi documenti digitalizzati suddivisi per tematiche, l'Università di Padova raccoglie le sue collezioni digitali - tanto archivistiche quanto museali - nel progetto phaidra [3] e l'Università "Federico II" di Napoli rende accessibili documenti di archivio, manoscritti, archivi fotografici e beni artistici e architettonici [2]; retemuseiuniversitari [4] propone una collaborazione attiva tra molte Istituzioni per definire il ruolo dei musei di natura scientifica, arricchendo con un alto livello di competenze i curricula scolastici e i percorsi di vita e carriera degli studenti. Ampliando lo sguardo verso il panorama internazionale, segnaliamo le gestioni innovative del patrimonio museale e archivistico universitario nelle collezioni online del Museum der Universität Tübingen [5], il cui sistema museale accoglie le collezioni più ampie della Germania. Una importante sezione è dedicata alla collezione di oggetti matematici [Seidl et al. 20 I 8; Pavignano et al. 2020; Pavignano, Zich 2020]. Anche se nella contemporaneità la produzione e l'uso didattico di modelli fisici parrebbe superato, è indiscusso il ruolo assunto nel tempo, che diviene ancora più fondamentale se associato all'epistemologia stessa che li generò. Ne consegue che un supporto descrittivo delle valenze semantiche degli oggetti matematici, che ne espliciti i parametri rispetto ai quali poter 
riconoscere nei modelli le rappresentazioni materiali di concetti matematici, sia necessariamente esito di mediazione tra valenze comunicative e didattiche. I modelli ottocenteschi, per esempio, pur nella loro semplicità materiale, si configurano come artefatti visuali, in quanto illustrazione tangibile di risultati teorici [Gay 2000, pp. 43-52] e perciò si presentano come connessione con il sistema astratto di segni.

\section{Per un nuovo Catalogo: quesiti geometrici e intenzioni comunicative}

La nostra collezione di modelli si confronta con l'intenzione di recuperare l'uso didattico di oggetti tangibili e di incentivarne la produzione in proprio. I cataloghi di riferimento elencavano le proprietà geometriche del singolo modello con una breve descrizione testuale, qualche indicazione analitica e solo raramente una rappresentazione grafica [Pavignano et al. 2020]. I nostri artefatti sono stati ideati con il fine di essere prodotti e divulgati attraverso le sintetiche informazioni inserite in un catalogo visuale sistematizzato -tecnico e divulgativo- non più un catalogo per la vendita; catalogo che, oltre a divulgare, consente di percorrere la produzione con un certo criterio prestabilito [Innocenti 2007] e un linguaggio comune, mediazione tra l'uso di un linguaggio specifico e rigoroso che ottimizzi il transito informativo e uno divulgativo che ne permetta la traduzione trasversale. Da una parte, il concetto di linguaggio in Matematica è usato con varie accezioni, anche se in questi ultimi anni ha assunto un significato ampio, si veda l'inquadramento teorico del semiotic bundle [Arzarello et al. 2009], includendo parole e simbolismo, diagrammi, gesti e interazione con artefatti; pur senza addentrarsi nella questione, si può osservare che indubbiamente il linguaggio riveste un ruolo importante nella disciplina, sia che si identifichi il pensiero matematico con il discorso e la comunicazione [Sfard 2008] sia che ci si attenga all'esistenza indipendente degli oggetti matematici come realtà oggettiva di cui non è possibile fare

\begin{tabular}{|c|c|c|}
\hline \multirow[t]{2}{*}{ serie } & \multirow[t]{2}{*}{00} & Modelli base origami e kirigami. Edifici \\
\hline & & Basic origami and kirigami models. Buildings \\
\hline \multirow[t]{2}{*}{ serie } & 01 & Modelli origami. Superfici voltate esito di intersezione di cilindri \\
\hline & & Origami models. Vaulted surfaces generated by cylinders \\
\hline \multirow[t]{2}{*}{ serie } & 02 & Strumenti per la divulgazione scientifica. Reggia di Venaria. I/ Re e l'Origami \\
\hline & & Tools for scientific dissemination. Royal Residence of Venaria. The King and the origami \\
\hline \multirow[t]{2}{*}{ serie } & 03 & Visualizzazione del costruito. Reggia di Venaria. II Re e l'Origami \\
\hline & & Built visualization. Royal Residence of Venaria. The King and the origami \\
\hline \multirow[t]{2}{*}{ serie } & 04 & Approcci alla forma costruita. Mole Antonelliana \\
\hline & & Approaching built architecture. Mole Antonelliana \\
\hline \multirow[t]{2}{*}{ serie } & 05 & Modellazione origami. Mole Antonelliana \\
\hline & & Origami modeling. Mole Antonelliana \\
\hline \multirow[t]{2}{*}{ serie } & 06 & Tavoletta grafica. Strumenti per la comprensione della forma architettonica \\
\hline & & Graphic tablet. Tools to understand of the architectural shapes \\
\hline \multirow[t]{2}{*}{ serie } & 07 & Modellazione OriKirigami. Architettura costruita \\
\hline & & Orikirigami modeling. Built Architecture \\
\hline \multirow[t]{2}{*}{ serie } & 08 & Generalizzazione di quesiti geometrici relativi a sistemi di copertura a falde \\
\hline & & Generalization of geometric questions about pitched roofing systems \\
\hline \multirow[t]{2}{*}{ serie } & 09 & Comunicazione del costruito. Geometrie rilevate, geometrie modellate \\
\hline & & Built comunication. Surveyed and modeled geometries \\
\hline \multirow[t]{2}{*}{ serie } & 10 & Comunicazione di superfici teoriche \\
\hline & & Communication of theoretical surfaces \\
\hline \multirow[t]{2}{*}{ serie } & 11 & Laser cut. Poliedri \\
\hline & & Laser cut. Polyhedral \\
\hline \multirow[t]{2}{*}{ serie } & 12 & Laser cut. Sistemi di copertura \\
\hline & & Laser cut. Roofing systems \\
\hline
\end{tabular}




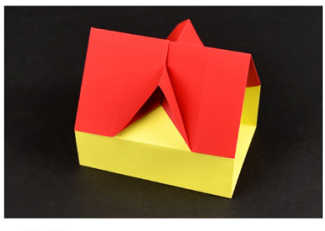

0.02 .01
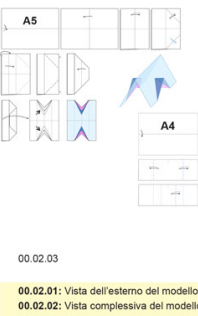

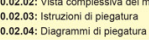

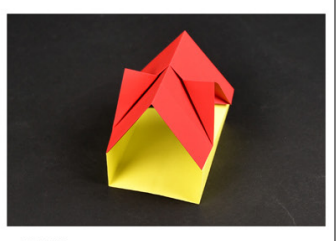

00.02 .02
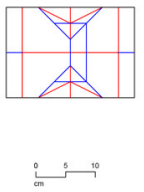

00.02 .04

00.02.01: Exterior vive of the mode
00.022.02: View of the mode

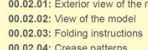

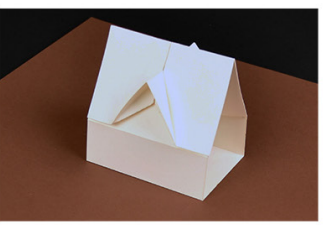

08.02 .01

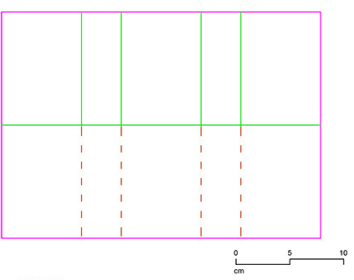

08.02

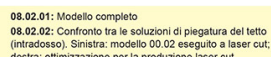

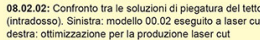

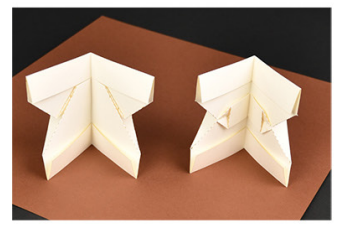

08.02 .02

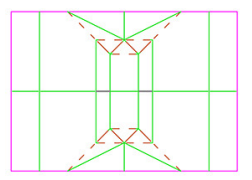

03.02.01: : vodel

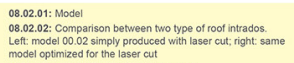

esperienza se non indirettamente [Duval 2006]. In ogni caso, come evidenziato da Maier ( 1989), l'uso adeguato del linguaggio rappresenta un mezzo potente di costruzione concettuale. Nel Catalogo proponiamo quindi che i termini tecnici vengano acquisiti attraverso l'uso di alcuni modelli illustrativi di un dato quesito geometrico, per poi essere ripresi nelle descrizioni successive. L'associazione di descrizioni testuali-grafico-analitiche permette di creare intersezioni utili a costruire un linguaggio condiviso su differenti registri della comunicazione e della conoscenza. Sistematizzando il corpus documentario dei modelli di superfici geometriche prodotto in questi anni abbiamo rispettato la cronologia di ideazione creando accorpamenti tematici e definito un indice ragionato di serie omogenee per caratteristiche geometriche della produzione e/o della finalità (tab. I). I modelli sono quindi descritti attraverso una serie di dati univoci che ne permettono la consultazione rapida, nell'intenzione di una loro riproducibilità in autonomia. Tra le informazioni, sono esplicitate le fasi di ideazione, elaborazione e stesura finale del progetto di produzione oltre alla sua descrizione comprensiva di indicazioni puntuali: fonti e contesti, modelli di ispirazione e ottimizzati. Ad esempio, la serie 00 presenta modelli di edifici come sequenza di pieghe e/o tagli, ottenuti senza strumenti per la produzione, mentre la serie 08 offre modelli esemplificativi del processo progettuale esito della mediazione tra l'approccio origamistico classico e quello laser cut attraverso la generalizzazione del processo geometrico costruttivo che si

Fig. 4. Estratto della scheda del modello 03.0 I, volta a botte su base rettangolare. Estratto della scheda del modello 03.02, porzione della Galleria Grande del Reggia di Venaria Reale (TO), dove si applica lo
schema di costruzione del modello 03.01.

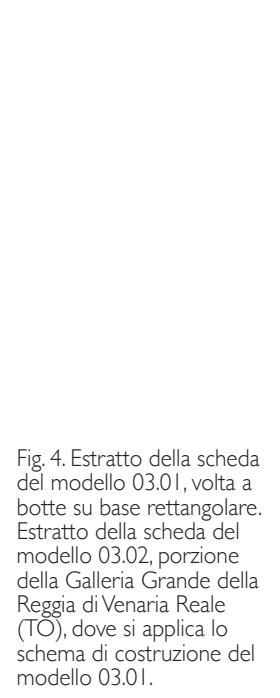

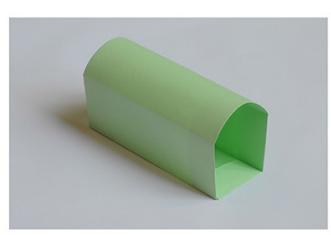

03.0101

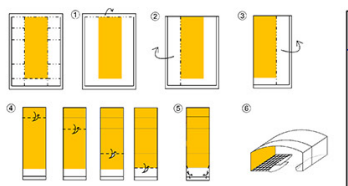

03.01.03

03.01.01: Veatua del mocello
03.01.02: Veatuta del modello

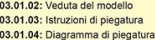

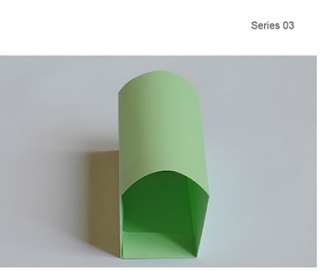

03.0.1.02

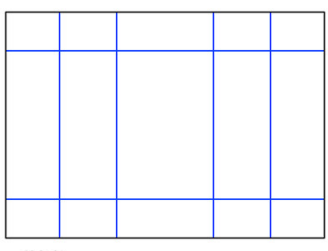

030104

03.01.01: Model verw
03.01.02: Model verw 03.01.02: Model ver
03.01.03: Folding instruction

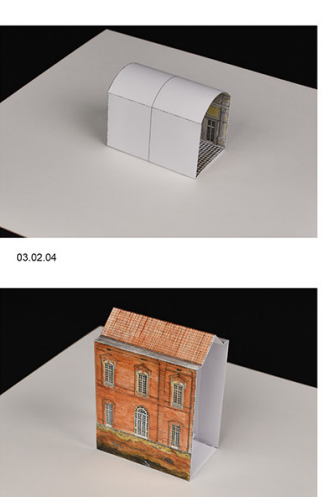

03.02 .06

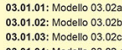
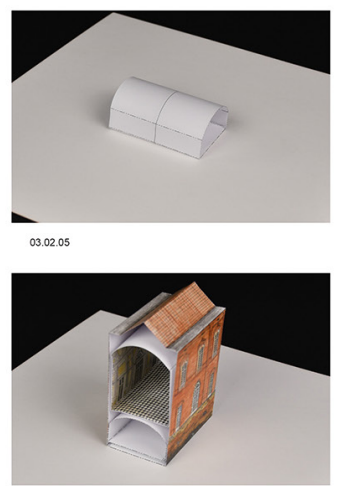

03.02 .0

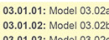
03.01.1.2: Model 1302202 
Fig. 5. Scheda di introduzione alle geometrie dei cilindri da intersecarsi per la generazione delle superfici teoriche rappresentate dai modell della serie 01, come il 01.01, volta a crociera con sezione catenaria.
Fig. 6. Supporti costruit per la 'messa in forma' dei modelli e per facilitarne le riprese fotografiche.

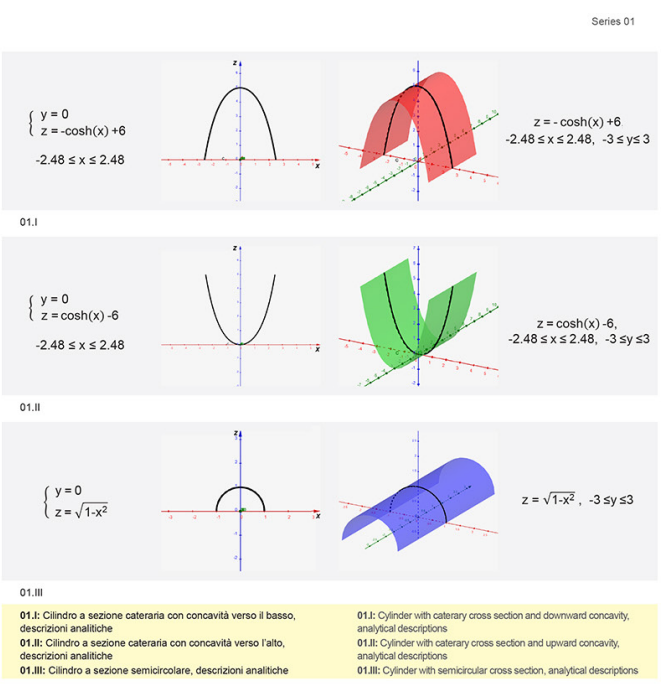

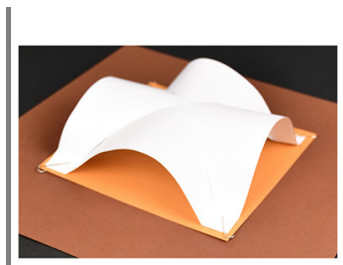

01.01 .01

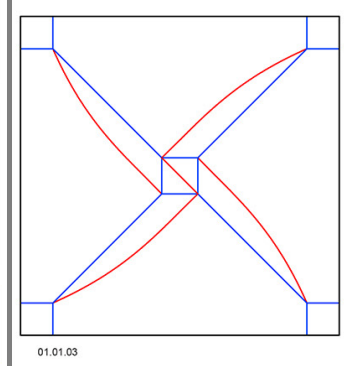

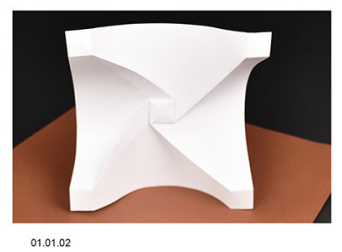

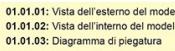

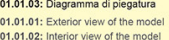
01.01.02: Interior view of the
01.01.03: Crease pattern svincola dalla forma reale per divenire strumento di visualizzazione di una forma teorica (fig. 3). Nella revisione dei materiali prodotti si è dato maggiormente spazio a modelli di forme architettoniche: oltre alla serie 00 , di riferimento a modelli origami già noti, undici delle dodici serie sono specifiche. I modelli catalogati sono artefatti iconici, idonei alla visualizzazione di architetture o parti delle stesse, qui ridotte ai minimi termini e private di ogni elemento all'infuori del proprio volume, in alcune occasioni addirittura lasciato inconcluso e definito solamente da intersezioni di superfici. In un crescendo di difficoltà, tutti i modelli offrono la possibilità di dare forma al volume attraverso poche operazioni e si possono quindi collocare tra i possibili ausili didattici per differenti età dello sviluppo, dallo stadio preoperatorio di Piaget ( 1955) al livello universitario. La serie 03 presenta un set di artefatti idonei a educare la mente anche alla discretizzazione dei dati da rappresentare: attraverso la modellazione fisica, il fruitore correla forma e rappresentazione superando i limiti di interpretazione della stessa. La sequenza di piega si avvale di un linguaggio grafico codificato supportato da rappresentazioni intuitive tanto da renderne l'uso proponibile sia in attività di didattica esperienziale sia in sede di formazione dei formatori [Spreafico, Zich 2017]. I modelli, in parte statici e in parte dinamici, sono pensati come oggetti riproducibili e smontabili. L'approccio origami permette di realizzare sia modelli simbolici sia modelli che rispettano il rigore geometrico delle superfici. Ad esempio, il modello 03.0 I rappresenta una volta a botte: può essere iconico, senza valenze geometriche, capace di cambiare sezione al variare della quantità di carta inserita nella tasca di base ma anche realizzato in modo rigoroso, per illustrare una geometria specifica nonostante la flessibilità del materiale cartaceo. La modellazione origami rende il modello adatto alla didattica esperienziale a differenti livelli e utile anche per introdurre le prime nozioni di linguaggio architettonico: qui, l'intersezione tra la superficie cilindrica e la parete verticale rappresenta il piano di imposta e evidenzia
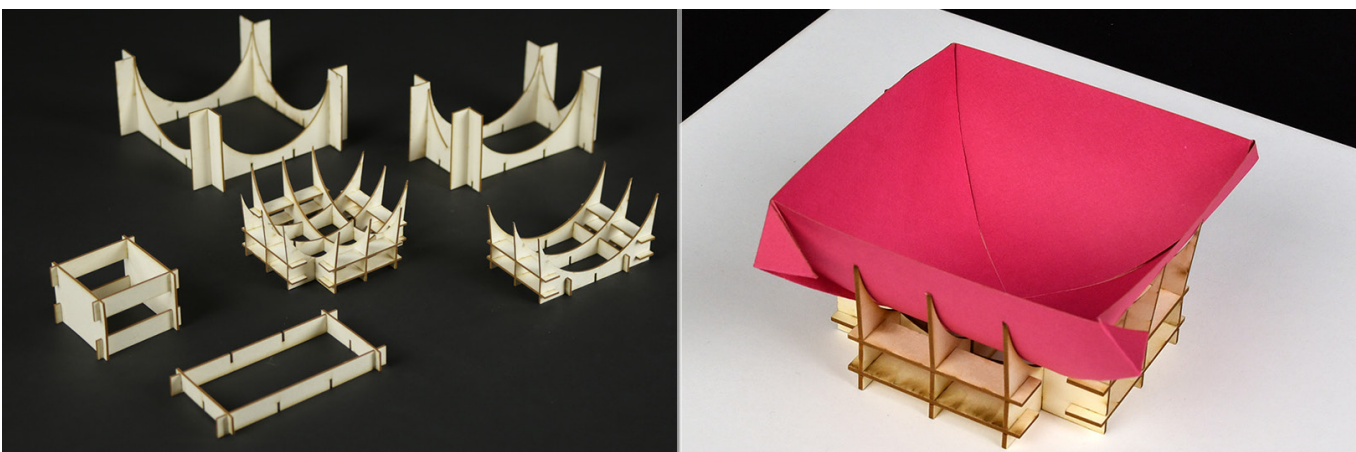
Fig. 7. Esempio di rasformazione di un modello di volta a

crociera: 01.01 , a sezione catenaria-origami; 03.07. sezione semicircolarerigami; a sezione semicircolare-laser cut.

Fig. 8. Schede relative a paraboloide iperbolico: I 0.01 , costruito per piani ezioni orizzontali-laser cut: 10.03 stampato in 3D; interazione visuale tra le differenti tipologie di prototipazione utili al miglioramento della comprensione e della percezione della forma teorica rappresentata dai

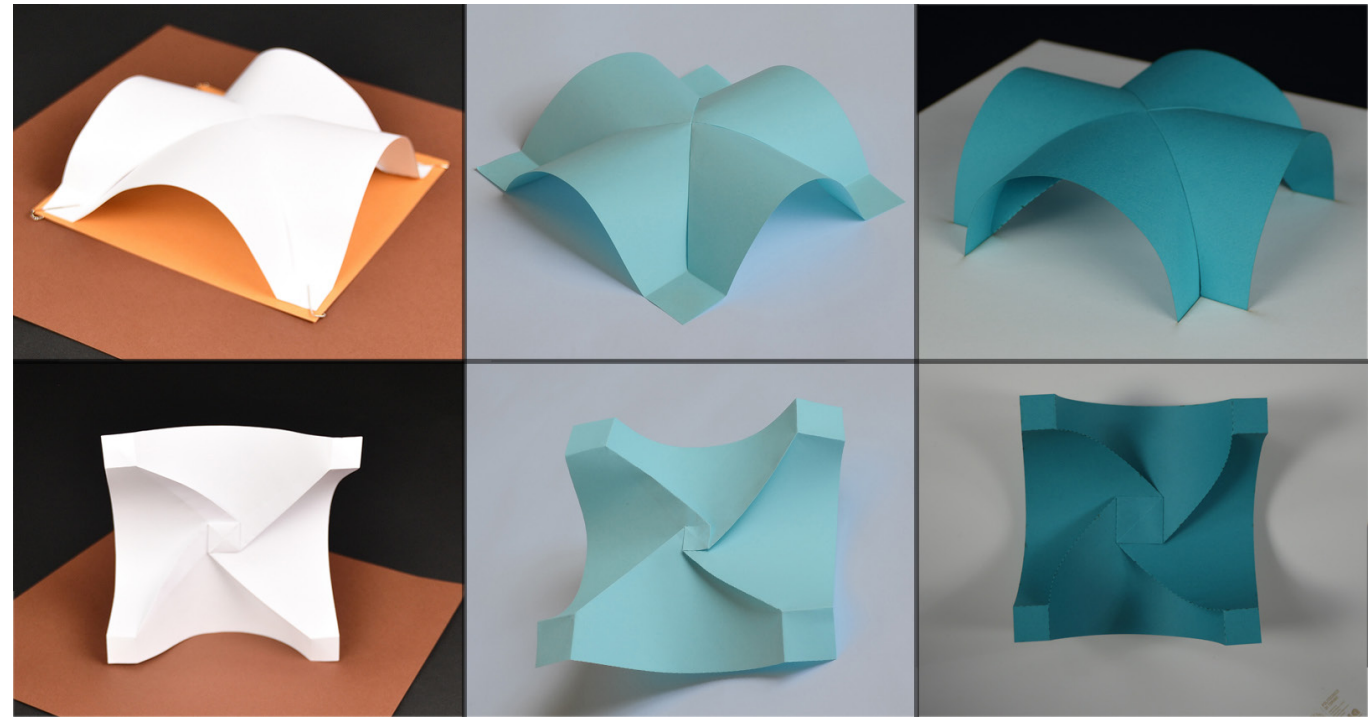

Serie 10 10.01 Paraboloide iperbolico (sella), piani sezione orizzontal
10.01 Hyperbolic paraboloid, horizontal cutting planes Idea | Concept
Progetto | Design
Modello | Model Tecnical Technique
Materiale | Material Materiale $\mid$ Mater
Dimensioni CP |
Fonti | Sources MP cartvegegale $2 \mathrm{~mm}$ | finn board $2 \mathrm{~mm}+\mathrm{MDF} 4 \mathrm{~mm}$ cartvegealale
varie |varies

I modello rappresenta una porzione della superficie
di equazione $z=x^{2} / a^{2}-y^{2} / b^{2}$ attraverso una model represents a portion of the surface piano $z=0$, e una coppia di rete, mentre ei intersezioni
con i pianni $z=k \neq 0$ sono iperboli le cui proiezioni sul
lines; on the other hand, intersections with horizontat

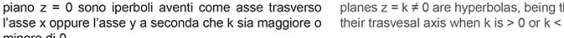
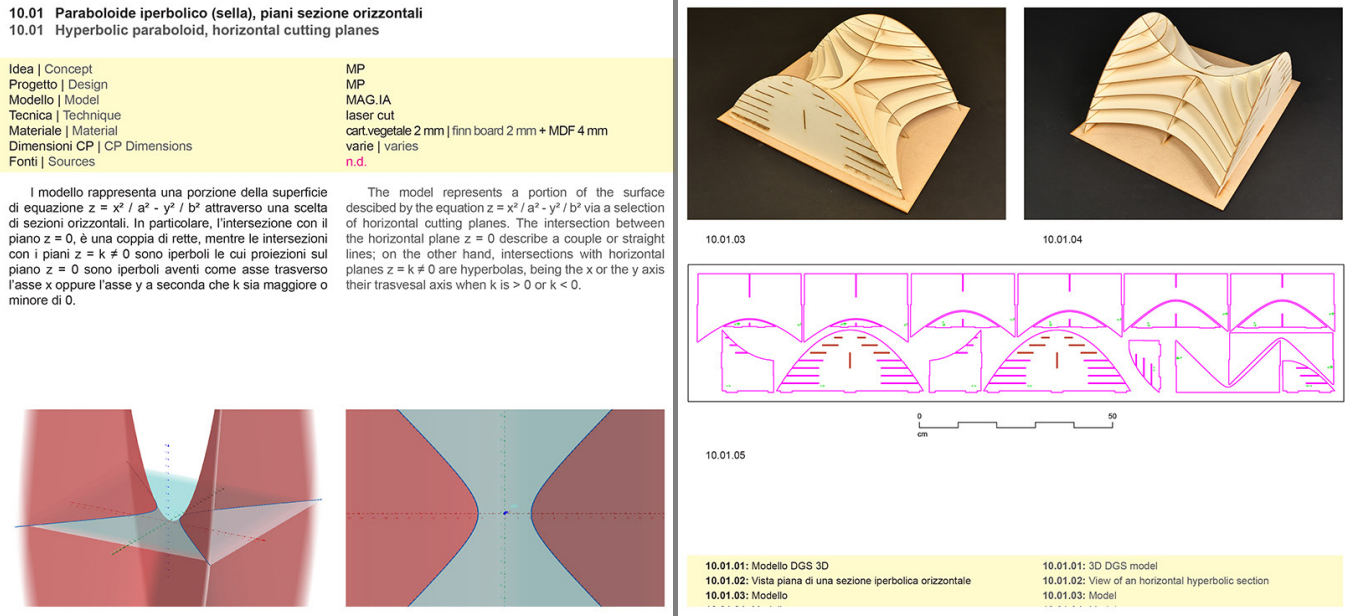

Series 10

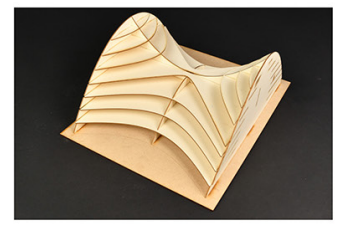

$$
\text { (1) }
$$


una retta che indica la direzione di traslazione dell'arco/curva direttrice; la linea di terra è definita dall'intersezione tra parete verticale e pavimento; la superficie interna è l'intradosso, quella esterna l'estradosso (fig. 4). La serie 0 I presenta differenti tipologie di volte generate dall'intersezione di due cilindri; la loro configurazione spaziale è l'esito di una prima ricerca di vincoli per poter controllare rigorosamente il processo geometrico di sviluppo delle superfici rigate (fig. 5). La serie 03 li rivisita variandone le geometrie di base e la serie 12 li ripropone cambiandone la tecnica di produzione, con processo di stampa laser cut, creazione di supporti per la 'messa in forma', elaborazione del supporto materico in modo da facilitarne la piega senza indebolirne la struttura (fig. 6) variandone accessibilità/riproducibilità e rigore geometrico. Per gestire al meglio le geometrie teoriche, nella serie $0 \mathrm{I}$ si presentano più soluzioni di uno stesso tipo di volta e ogni modello ha le proprie specificità comunicative esplicitate dalla gestione della carta in eccesso a sottolineare (o parzialmente nascondere) potenziali/limiti della rappresentazione plastica, mentre nella serie 12 il processo di ottimizzazione ha ridotto il numero delle possibili soluzioni a quelle migliori per lo strumento scelto (fig. 7). Nell'intenzione di valutare l'efficacia comunicativa di differenti modalità di produzione di uno stesso modello, la serie 10 confronta modelli ottenuti con la stampante 3D o come sequenza di piani sezione con la laser cutter utilizzata quindi non più per gestire superfici sviluppate, ma per definire uno scheletro sul quale leggere la pelle della forma da descrivere (fig. 8). La serie 09 riassume il processo di traduzione di una forma costruita, rilevata, in un modello fisico per trasmetterne le peculiarità geometriche a un fruitore non specialistico, sottolineando il dibattito intorno a quanti e quali siano i modelli utili a descrivere quale geometria per quale utente, per visualizzare la superficie teorica più plausibile per descriverne la complessità: il modello del costruito, con tutte le sue irregolarità non aiuterebbe infatti a cogliere le specificità geometriche della superficie (fig. 9). Molti sono i modelli che presentano il rapporto architettura costruita/modello fisico nella sua accezione iconica e pertanto non devono gestire tali irregolarità; in altri, l'architettura costruita è invece indagata attraverso la lettura critica delle fonti grafiche e il conseguente riconoscimento delle superfici teoriche ad essa più affine.

Fig. 9. Dalla forma reale a modello tangibile. Volta di Benedetto Alfieri, Reggia di Venaria Reale (TO).
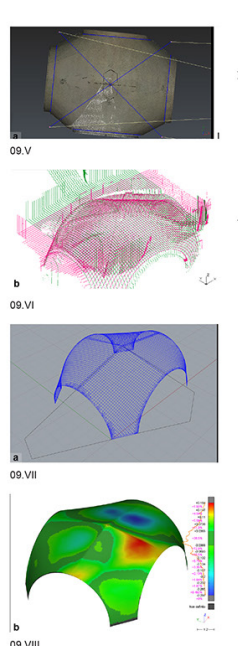

co.VIII

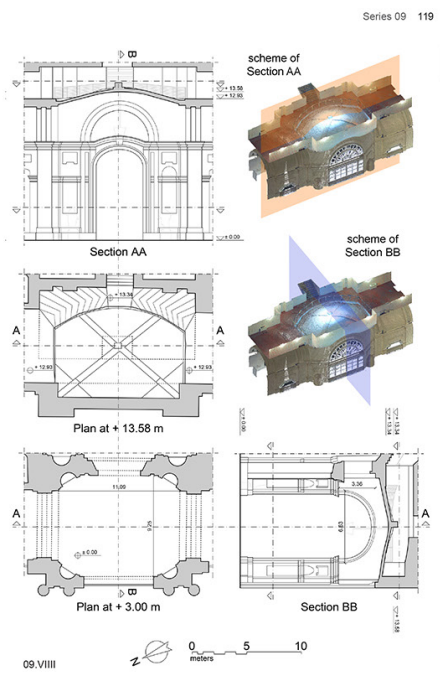

新

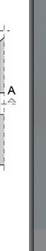

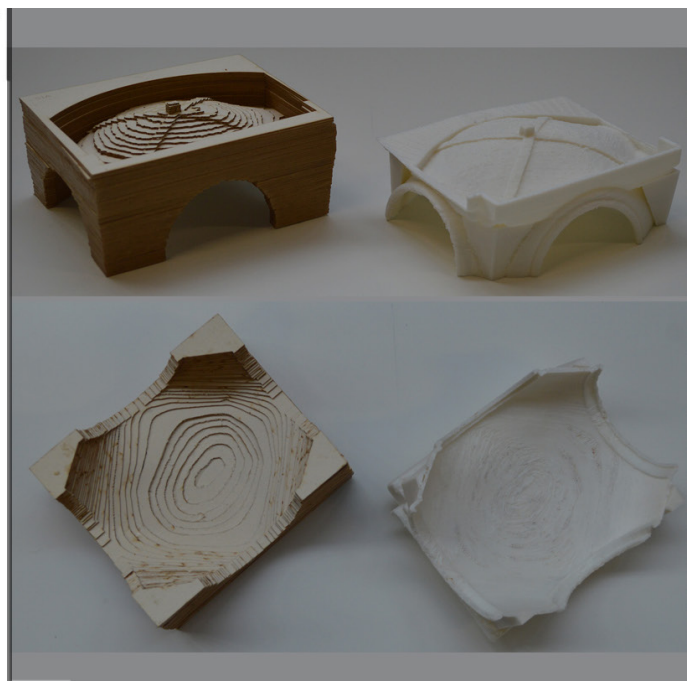

\section{Conclusioni}

La rilettura critica di alcuni cataloghi di superfici matematiche ha ispirato la stesura di un catalogo visuale di modelli esito di un percorso progettuale dettato dalla calibrazione dei contenuti in funzione del processo produttivo e della sua attitudine alla condivisione dei concetti in funzione delle differenti intelligenze coinvolte. I modelli sono evidentemente simbolici, rappresentazioni senza spessore delle superfici teoriche che costituiscono la strut- 
tura di forme architettoniche, presentati con un apparato grafico descrittivo e progettuale che li rende condivisibili. Le tecniche di produzione spaziano dal classico origami basato su sequenza di piega all'uso di laser cutter che imprime direttamente tutte le pieghe sul foglio, dalla stampa 3D alla modellazione con software di Geometria Dinamica (GeoGebra). Nel catalogo si sottolineano le intenzioni comunicative oltre a quelle geometrico descrittive e si propone il confronto tra modelli di una stessa forma risolti con tecniche e approcci differenti per educare alla lettura della forma architettonica. II rapporto superficie teorica/modello fisico/architettura costruita, sia essa rilevata direttamente o interpretata attraverso la lettura critica di fonti di progetto o di rilievo, trova spazio nel catalogo nel rispetto delle finalità dello stesso: la possibilità di accompagnare teoricamente e operativamente alla produzione di modelli con differenti registri comunicativi. Tutti i modelli del catalogo sono strumenti idonei per educare la mente alla percezione dei volumi e alla discretizzazione dei dati da rappresentare, pertanto possono essere proposti dalla docenza in differenti momenti e contesti didattici, a più livelli. Nelle sempre più attuali dinamiche della didattica a distanza, la sperimentazione di attività laboratoriali diffuse ci ha dato conferme sulla loro efficacia.

\section{Ringraziamenti}

Ringraziamo il ModLab Architettura (DAD - PoliTo) per il supporto alla prototipazione, il RilDoc (DAD - PoliTo) per il supporto alla riproduzione fotografica, tutti gli studenti che sono intervenuti dal 2015 a oggi nell'ambito dei progetti di ricerca che hanno portato alla realizzazione dei modelli presentati.

C. Cumino è membro del Gruppo Nazionale per le Strutture Algebriche, Geometriche e le loro Applicazioni (GNSAGA-INDAM). Per M. Pavignano e U.Zich il contributo è stato sviluppato nell'ambito del progetto di ricerca MAG.IA 2020: Matematica Architettura Geometria. Interconnessioni Applicative.

\section{Note}

[I] <https://sbs.uniromal.it/sapienzadigitallibrary> (consultato il 20 febbraio 202I).

[2] <www.eco.unina.it> (consultato il 20 febbraio 202I).

[3] <www.phaidra.cab.unipd.it> (consultato il 20 febbraio 202I).

[4] <www.retemuseiuniversitari.unimore.it> (consultato il 20 febbraio 2021).

[5] <www.unimuseum.uni-tuebingen.de> (consultato il 20 febbraio 202I).

\section{Riferimenti bibliografici}

Cardone V. (20 | 6). Imagining the cultural area of the visual images. In XY digitale, I (I), pp. I2-27.

Cumino C. et al. (2017). Modelli cartacei per la comprensione della forma architettonica: ricerca, progetto, sperimentazione e didattica in un dialogo tra geometria e rappresentazione. In A Di Luggo et al. (a cura di). Territori e frontiere della rappresentazione. Atti del $39^{\circ}$ Convegno Internazionale dei Docenti delle Discipline della Rappresentazione. Napoli, I4- I 6 settembre 2017, pp. 1279- 1286. Roma: Gangemi.

Cumino C. et al. (2020). Geometry to Build Models, Models to Visualize Geometry. In Digital Experiences in Mathematics Education, pp. 149-166.

De Fusco R. (20।0). Architecturminimum: Le basi dello storicismo, strutturalismo, semiotica, ermeneutia \& altre teorie. Napoli: Clean Edizioni.

DickW. ( 1892). Katalog mathematischer und mathematisch-physikalischer Modelle, Apparate und Instrumente. München: K. Hof. u. Universitäts Buchdruckerei von Dr. C. Wolf \& Sohn.

Duval R. (2006). A cognitive analysis of problems of comprehension in the learning of mathematics. In Educational Studies in Mathematics, 61 ( I-2), pp. I03-131.

Emch A. (1920). Mathematical models. In University of Illinois Bulletin, XVIII ( I2).

Emch A. (1923). Mathematical models II series. In University of Illinois Bulletin, XX (42).

Emch A. (1925). Mathematical models III series. In University of Illinois Bulletin, XXII (35).

Emch A. (1928). Mathematical models IV series. In University of Illinois Bulletin, XXV (43).

Estanave E. (s.d.). Construction des modèles de surfaces applicables sur le paraboloide de révolution dèfinies par M. G. Darboux. Paris: Gauthier-Villars. 
Fabre de Lagrange M., Merrifield C.W. (1872). A catalogue of a collection of models of ruled surfaces. London: George E. Eyre and William Spottiswoode.

Fehr H. F. (1938). The construction and use of mathematical models. Upper Montclair: s.e.

Gay F. (20 I7). Immagini che parlano di immagini. In A. Luigini (a cura di). Lineis Describere. Sette seminari tra rappresentazione e formazione, pp. 94- I 17. Melfi: Libria.

Gay F. (2000). Intorno agli Omolografi. Strumenti e Modelli per la Geometria Descrittiva. Venezia: IUAV.

Giacardi L. (20I5). Geometric Models in Mathematics Teaching in Italy at the Turn of the Twentieth Century. In Mathematisches Forschungsinstitut Oberwolfach, 47, pp. 2784-2787.

Guarini G. (1737). Architettura civile. Torino: Gianfrancesco Mairesse.

Innocenti P. (2007). A proposito di Bucherkunde: I. La Bibliographia di Blum, 2. II Fra biblioteca e archivio di Cavallaro, 3. Due recensioni di Serrai: una risata le seppelli. In Culture del testo e del documento, n. 25, pp. 27-70.

Maier H. (1989). Conflit entre langue mathématique et langue quotidienne pour les élèves. In Cahiers de didactique des mathématiques, 3, pp. 86-1 18.

Neuendorff R. (1922). Praktische mathematik. Leipzig: B. G. Teubner.

Pavignano M., Cumino C., Zich U. (2020). Catalog Mathematischer Modelle: Connessioni tra testo, rappresentazione grafica e descrizione analitica. In A. Arena et al. (a cura di). Connettere. Un disegno per annodare e tessere. Atti del $42^{\circ}$ Convegno internazionale dei Docenti delle Discipline della Rappresentazione, pp. 3660-3677. Milano: Franco Angeli.

Pavignano M., Zich U. (2020). Colore tra forma e materia dei modelli fisici per lo studio della Geometria. In V. Marchiafava, M. Piccolo (a cura di). Colore e Colorimetria. Contributi Multidisciplinari, vol. XVI A, pp. 398-405. Milano: Gruppo del Colore Associazione Italiana Colore.

Quaroni L. (1978). Progettare un edificio: Otto lezioni di architettura. Milano: Mazzotta.

Schilling M. (1903). Catalog mathematischer Modelle für den höheren mathematischen Unterricht. Halle: Martin Schilling.

Schilling M. (191 I). Catalog mathematischer Modelle für den höheren mathematischen Unterricht. Leipzig: Martin Schilling.

Sdegno A. et al. (20 17). Modellare smorfie. Rilievo e rappresentazione aptica di due teste scultoree di Franz Xavier Messerschmidt. In A Di Luggo et al. (a cura di). Territori e frontiere della rappresentazione. Atti del $39^{\circ}$ Convegno Internazionale dei Docenti delle Discipline della Rappresentazione. Napoli, I4- 16 settembre 20 17, pp. 969-976. Roma: Gangemi.

Seidl E., Loose F., Bierende. E. (ed.) (20।8). Mathematik mit Modellen. Alexander von Brill und die Tübinger Modell Sammlung. Tübingen: Museum der Universität Tübingen.

Sfard A. (2008). Thinking as communicating: Human development, the growth of discourses, and mathematizing. Cambridge University Press.

Spreafico M. L., Zich U. (20 I7). Train the trainers on learn geometry by doing. In: L. Gómez Chova, A. López Martínez, I. Candel Torres (a cura di). EDULEARN 1 7. Proceedings 9th International Conference on Education and New Learning Technologies. Barcelona 3-5 July 2017, pp. 1969-1976. Valencia: IATED.

Stoll R. (|96I). Lehrmodelle für Mathematik. Berlin: Rudolf Stoll K.G.

Tagliari A., Florio W. (2013). Digital fabrication of physical models to analyze unbuilt projects using laser cutter. In P. J. da Silva Bartolo et al. (a cura di). High Value Manufacturing: Advanced Research in Virtual and Rapid Prototyping. Proceedings of the 6th International Conference on Advanced Research in Virtual and Rapid Prototyping. Leiria, Portugal, I-5 October 20 I 3. Boca Raton (FL): CRC Press.

Wiener H. (1907). Abhandlungen zur Sammlung mathematischer Modelle. Leipzig: B. G. Teubner.

Wiener H. ( I9II). Abhandlungen zur Sammlung mathematischer Modelle. Leipzig: B. G. Teubner.

Wiener H., Treutlein, P. (1912). Verzeichnis mathematischer Modelle. Leipzig: B. G. Teubner.

\section{Autori}

Caterina Cumino, Politecnico di Torino, caterina.cumino@polito.it

Martino Pavignano, Politecnico di Torino, martino.pavignano@polito.it

Ursula Zich, Politecnico di Torino, ursula.zich@polito.it

Per citare questo capitolo: Cumino Caterina, Pavignano Martino, Zich Ursula (202I). Proposta di un catalogo visuale di modelli per lo studio della forma architettonica tra Matematica e Disegno Nisual catalog of models for the study of architectural shapes between Mathematics and Drawing: a new proposal. In Arena A Arena M. Mediati D. Raffa P (a cura di). Connettere. Un disegno per annodare e tessere. Lingugogi Distanze TecnolDistances Technologies. Proceedings of the $42^{\text {th }}$ International Conference of Representation Disciplines Teachers. Milano: Franco Angeli, pp. 626-645.
Diting 


\title{
Visual Catalog of Models for the Study of Architectural Shapes between Mathematics and Drawing: a New Proposal
}

\author{
Caterina Cumino \\ Martino Pavignano \\ Ursula Zich
}

\section{Abstract}

A critical re-reading of late 19th/early 20th century catalogs of mathematical surfaces proved to be a source of inspiration for the exploration of Geometry as a shared language to investigate Architecture and for a systematization of tangible models born as training/educative aids for understanding and representation of the architectural form.

The creation of a descriptive and design graphic apparatus that makes these models accessible, through a visual catalog is an outcome of the more complex interdisciplinary research project MAG. IA, Mathematics, Architecture, Geometry. Application and Interconnections.

The detailed analysis of some of the aforementioned historical catalogues also highlighted the great relevance of this approach when the dematerialization of many teaching aids, in direct correlation to the need to offer training remotely, pushes towards a massive digitalization to the detriment of a manual and tangible practice that is implicit in the production of physical models.

\section{Keywords}

catalogue, physical models, geometry, representation, architectural shape.

Models of surfaces, examples of catalogues: Katalog mathematischer und mathematisch-physikalischer Modelle [Dick I892, p. 286]; authors proposal discussed in the contribution.

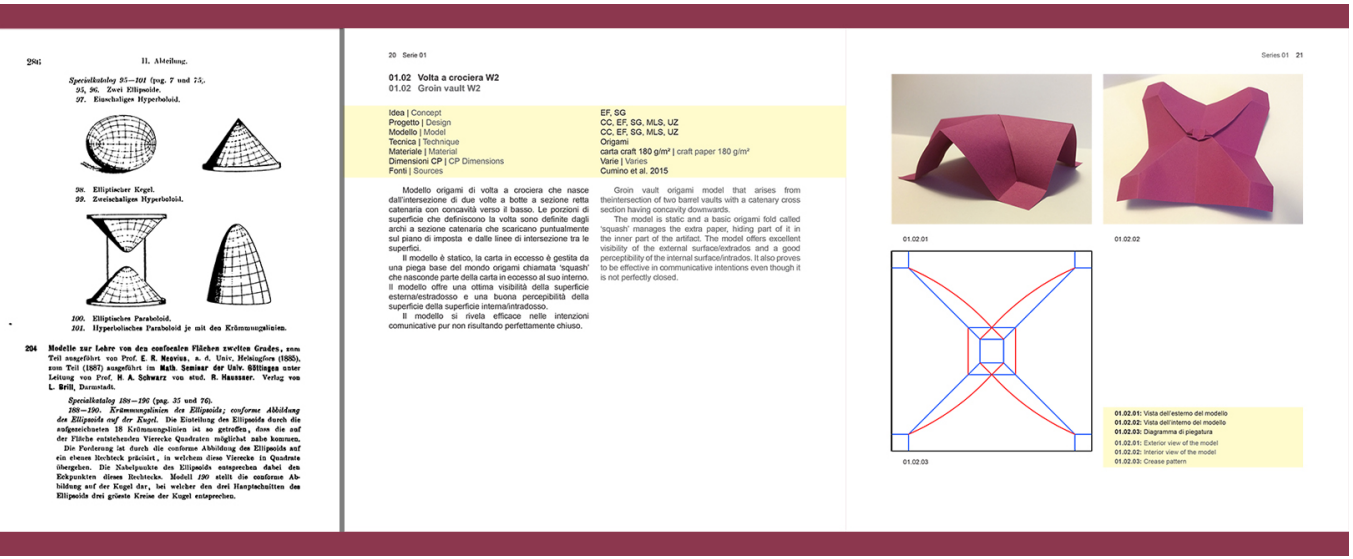




\section{Introduction}

Geometry is a common topic between Mathematics and Architecture: in the first, it deals with abstract spaces, while in the second one it concerns with concrete space [Quaroni 1978] and "Architecture relies on Geometry, thus he [the architect] needs to know at least its basic principles" [Guarini 1737, p. 3]. Over centuries, Geometry developed increasingly refined and articulated methods and models, which can be described by means of various languages, which correspond to different specializations. Some of them are of more interest to the architect, from an applicative point of view; for example, Synthetic Geometry analyses objects in the Euclidean plane and space from the point of view of the process underlying their construction is perhaps the most congenial to the mindset of architects as it can communicate with their main language, Drawing [De Fusco 20I0, Cardone 2016] and, as such, is the basis of Descriptive Geometry. In Europe during the nineteenth century and precisely in the academic context of Descriptive Geometry, a great interest arose in the production of tactile and visual aids for the practical teaching of theory. World fairs and exhibitions from London I85 I to Saint Louis 1904- were contexts where mathematical instruments, models and machines for calculation and drawing were exhibited to the public. They were occasions to spread a material culture in a geopolitical dimension that born around mathematical objects, a context where the mathematical community promoted exchanges of ideas and technology [Giacardi 2015] and beyond, since the first Schilling catalog (1903) introduced some models of architectural objects (fig. I) useful for developing coping skills from still-life and for recognizing the constituent geometries [Wiener I9II]. Nonetheless, designers were also sellers and their catalogues of models of mathematical surfaces were tools for promotion, later becoming a tangible memory of the cultural fervour underpinning models production. Hermann Wiener, professor of Descriptive Geometry in Karlsruhe, became known for the mathematical models he presented at exhibitions as aids for teaching mathematics [Wiener 1907, p. 3]. Today, those objects, which have become property of Institutions and Museums collections, are often not very accessible, usually mediated by the dynamics of online use that influence their essence by offering an indirect experience of a tangible product. On the other hand, beyond the effect of 'wunderkammer curiosity'

Fig. I. Examples of visual/ textual descriptions from the Catalog mathematischer Modelle by Martin Schilling (1903): VI.
Infinitesimalgeometrie de Flächen (p. 103); VIII. Modelle zur darstellenden Geometrie, zur darstelle

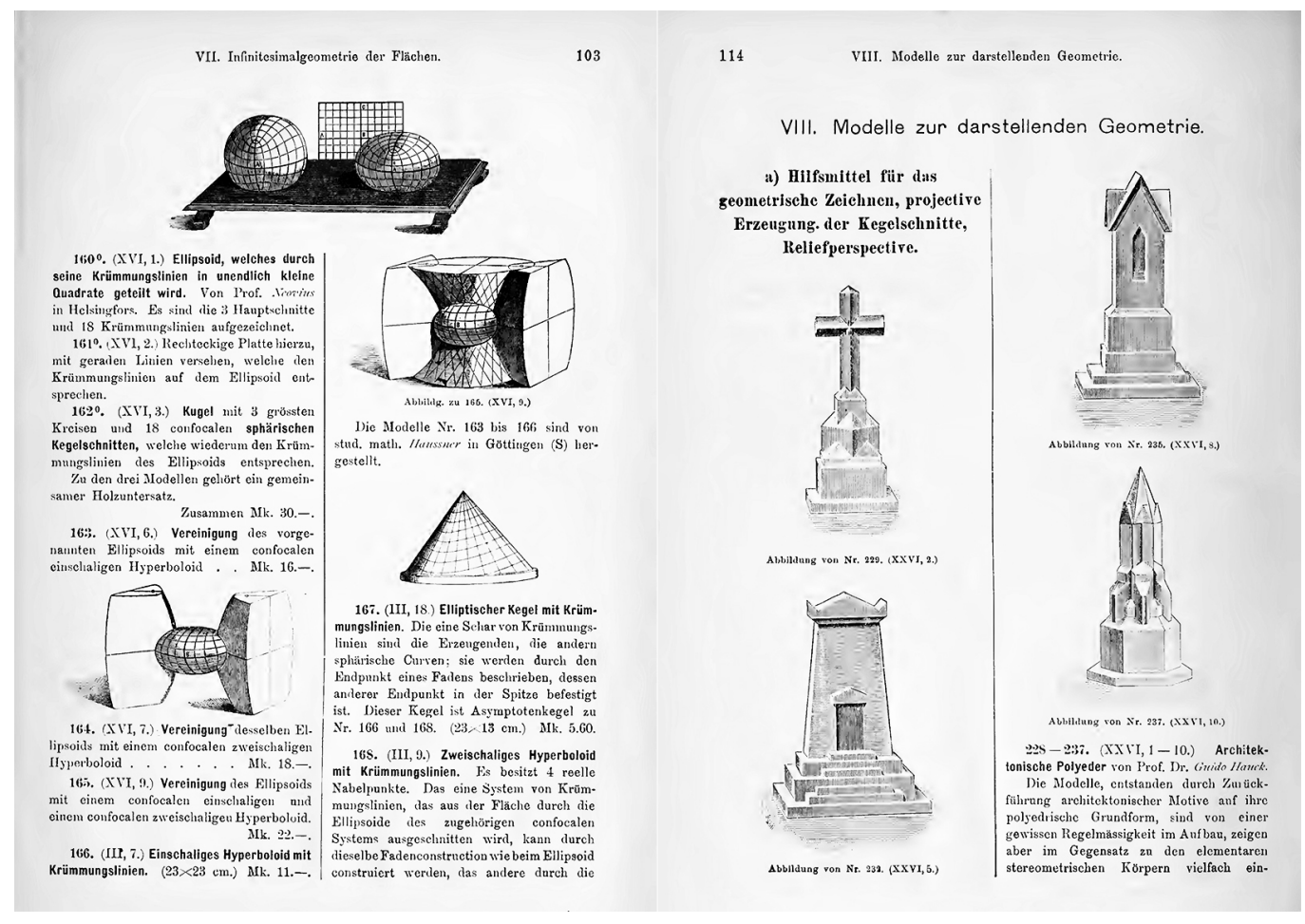


Fig. 2. Critical re-reading of some catalogues of mathematical models, visualization of covers, from 1872 to $|96|$. Fabre de Lagrange, Merriefiled (1872), Dick (1892) Schilling (1903), (1892), Schilling (1903), (1911), (1907), Schilling (1911), Wiener (1911), Wiener, Treutlein (1912), (1920-1928), Stoll (1961) they can arouse in the contemporary public, they still seem worthy of attention from the scientific community, not so much for their purely mathematical prerogatives, but rather for their characteristic of visual artifacts [Gay 20 17, p. 95] useful for formulating a sort of visual language of Geometry. In this sense, exploring the relationships between Mathematics and Drawing to identify ways of understanding and representing architectural shapes, we have developed over the years methodologies that rely on the physical model because of the mediation between the two cultural/scientific approaches. A critical re-reading of catalogues of mathematical surfaces of the late nineteenth/early twentieth century was a source of inspiration for us, specifically for a systematization of the artifacts produced up to now and for the creation of a descriptive and design graphic apparatus that made these models accessible, through a visual catalogue. The detailed analysis of these repertoires has highlighted the great relevance of this approach at a time when the need for remote teaching leads to the dematerialization of many teaching aids to the detriment of the tangibility implicit in the production of physical models (fig. 2).
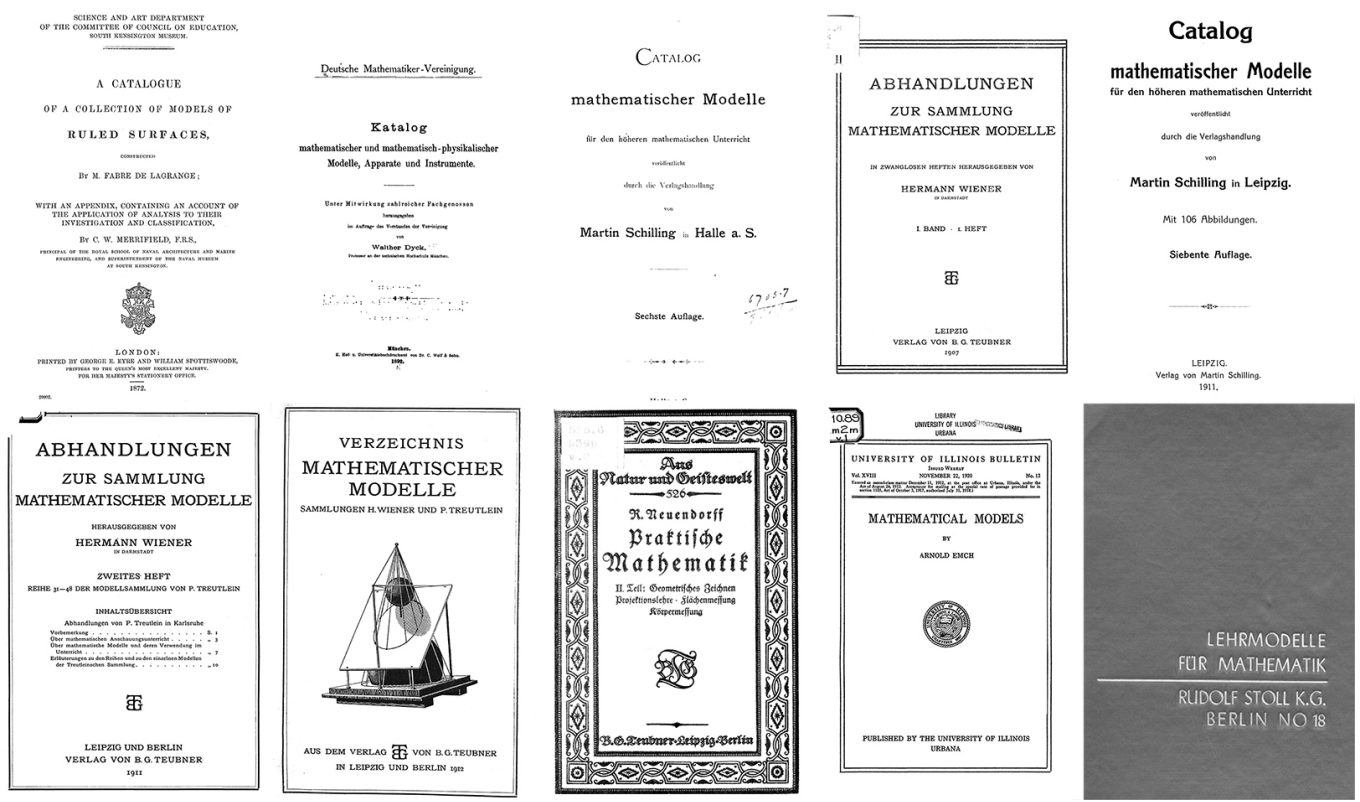

\section{Scientific collections on the web}

In Italy many scientific collections are variously exhibited on the web. Among the Institutes that explore the potential of digital approaches we find: the "La Sapienza" University of Rome [I] which provides numerous digitized documents divided by theme, the University of Padua that gathers its digital collections - both archival and museum - in the project phaidra [3] and the "Federico II" University of Naples which provides access to many archival documents, manuscripts, photographic archives and artistic/architectural heritage [2]. Moreover, the retemuseiuniversitari [4] proposes an active collaboration between many institutions to better define the role of museums of a scientific nature, enriching school curricula and life and career paths of students with a high level of scientific skills. Moving to the international panorama, we point out the innovative management of the university museum and archival heritage in the online collections of the Universität Tübingen [5], whose digital museum system houses the largest virtual collections in Germany. An important section is dedicated to tangible mathematical objects [Seidl et al. 20 I8; Pavignano et al. 2020; Pavignano, Zich 2020]. Although in contemporary times the production and educational use of physical models would seem outdated, the role they assumed over time is undisputed and it becomes even more fundamental when associated with the epistemology that generated them. Describing the semantic values of mathematical objects via properly designed 
aids helps in clarifying parameters with respect to which the material representations of mathematical concepts can be recognized in the models. Thus, this process is the result of mediation between communicative and educational values. The nineteenth-century models, for example, despite their material simplicity, are configured as visual artifacts, as a tangible illustration of theoretical results [Gay 2000, pp. 43-52] and therefore appear as a connection with the abstract system of signs.

\section{For a new Catalog: geometrical questions and communicative aims}

Our collection of models is born with the aims of recovering the educational use of tangible objects while encouraging their self-production. We started our project by analyzing historical Catalogs that used to list geometric properties of the single model with a short textual description, some analytical information and sometimes, but rarely, with graphic representation [Pavignano et al. 2020]. We designed our artifacts with the aim of being produced and disseminated through a set of synthetic information included in a systematized visual catalog -technical and informative- no longer a catalog for sale; a catalog that, in addition to disseminating, allows you to run through the production with a certain pre-established criterion [Innocenti 2007] and a common language, mediation between the use of a specific and rigorous language that optimizes information transit and a popular language that allows transversal translation. On the one hand, the concept of language in Mathematics is used with various meanings, although in recent years it has taken on a broad meaning, see the theoretical framework of semiotic bundles [Arzarello et al. 2009], including words and symbolism, diagrams, gestures and interaction with artifacts; even without going into the question, it can be observed that language undoubtedly plays an important role in the discipline, whether we identify mathematical thinking with speech and communication [Sfard 2008] or

\begin{tabular}{|c|c|c|}
\hline \multirow[t]{2}{*}{ serie } & \multirow[t]{2}{*}{00} & Modelli base origami e kirigami. Edifici \\
\hline & & Basic origami and kirigami models. Buildings \\
\hline \multirow[t]{2}{*}{ serie } & 01 & Modelli origami. Superfici voltate esito di intersezione di cilindri \\
\hline & & Origami models. Vaulted surfaces generated by cylinders \\
\hline \multirow[t]{2}{*}{ serie } & 02 & Strumenti per la divulgazione scientifica. Reggia di Venaria. /I Re e l'Origami \\
\hline & & Tools for scientific dissemination. Royal Residence of Venaria. The King and the origami \\
\hline \multirow[t]{2}{*}{ serie } & 03 & Visualizzazione del costruito. Reggia di Venaria. I/ Re e l'Origami \\
\hline & & Built visualization. Royal Residence of Venaria. The King and the origami \\
\hline \multirow[t]{2}{*}{ serie } & 04 & Approcci alla forma costruita. Mole Antonelliana \\
\hline & & Approaching built architecture. Mole Antonelliana \\
\hline \multirow[t]{2}{*}{ serie } & 05 & Modellazione origami. Mole Antonelliana \\
\hline & & Origami modeling. Mole Antonelliana \\
\hline \multirow[t]{2}{*}{ serie } & 06 & Tavoletta grafica. Strumenti per la comprensione della forma architettonica \\
\hline & & Graphic tablet. Tools to understand of the architectural shapes \\
\hline \multirow[t]{2}{*}{ serie } & 07 & Modellazione OriKirigami. Architettura costruita \\
\hline & & Orikirigami modeling. Built Architecture \\
\hline \multirow[t]{2}{*}{ serie } & 08 & Generalizzazione di quesiti geometrici relativi a sistemi di copertura a falde \\
\hline & & Generalization of geometric questions about pitched roofing systems \\
\hline \multirow[t]{2}{*}{ serie } & 09 & Comunicazione del costruito. Geometrie rilevate, geometrie modellate \\
\hline & & Built comunication. Surveyed and modeled geometries \\
\hline \multirow[t]{2}{*}{ serie } & 10 & Comunicazione di superfici teoriche \\
\hline & & Communication of theoretical surfaces \\
\hline \multirow[t]{2}{*}{ serie } & 11 & Laser cut. Poliedri \\
\hline & & Laser cut. Polyhedral \\
\hline \multirow[t]{2}{*}{ serie } & 12 & Laser cut. Sistemi di copertura \\
\hline & & Laser cut. Roofing systems \\
\hline
\end{tabular}


Fig. 3. Catalogues' files allow a comparison between different types of models (referred to the same object), here an example between models 00.02 and 08.02
Fig. 4. Detail of the file of model 03.0 I, barrel vault on a rectangular base. Detail of the file of model 03.02, portion of the Galleria Grande in the Royal Palace of Venaria Royal Pace of Venaria Reale (TO), where readers can analyze the scheme of 03.01

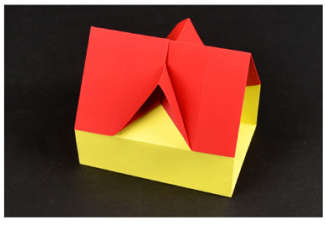

00.02 .01

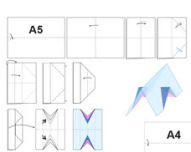

00.02 .03 00.02.01: Vista del esterno del modello
00.02.02: Vista complessiva del modell

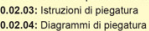

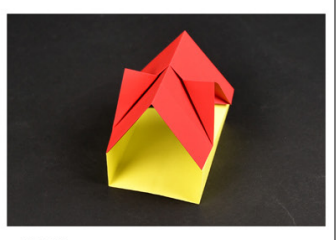

00.02 .02
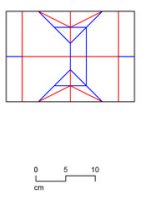

00.02 .04

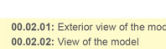

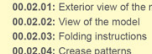

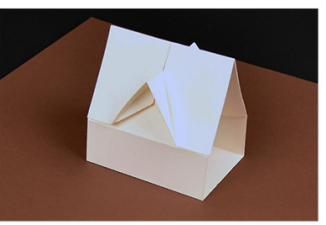

08.02 .01

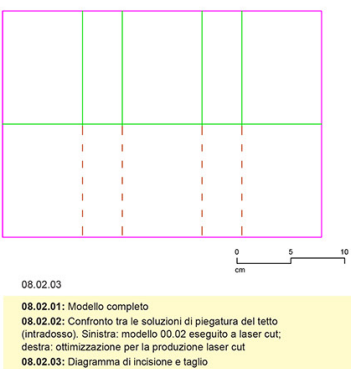

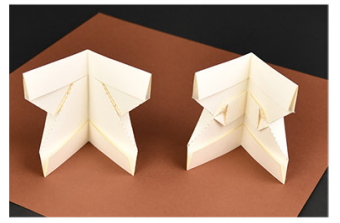

08.0202

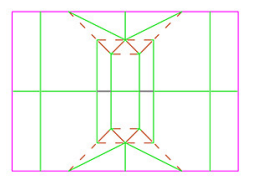

aseren:

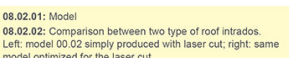

we adhere to the independent existence of mathematical objects as objective reality which cannot be experienced except indirectly [Duval 2006]. In any case, as highlighted by Maier [1989], the adequate use of language represents a powerful means of conceptual construction. In our Catalog, we therefore propose that the technical terms be acquired through the use of some illustrative models of a given geometric question, and then be taken up in subsequent descriptions. The association of textual-graphic-analytical descriptions allows you to create useful intersections to build a shared language on different registers of communication and knowledge. By systematizing the documentary corpus of geometric surface models produced in recent years, we have respected the chronology of conception by creating thematic groupings and defined a reasoned index of homogeneous series by geometric characteristics of production and / or purpose (table I). The models are therefore described through a series of univocal data that allow them to be consulted quickly, so that they can be autonomously reproduced. Among the information, the phases of conception, elaboration and final drafting of the production project are explained, as well as its description including precise indications: sources and contexts, inspiration and optimized models. For example, the 00 series presents models of buildings as a sequence of folds and / or cuts, obtained without tools for production, while the 08 series offers exemplary models of the design process resulting from the mediation between the classic origamistic approach and

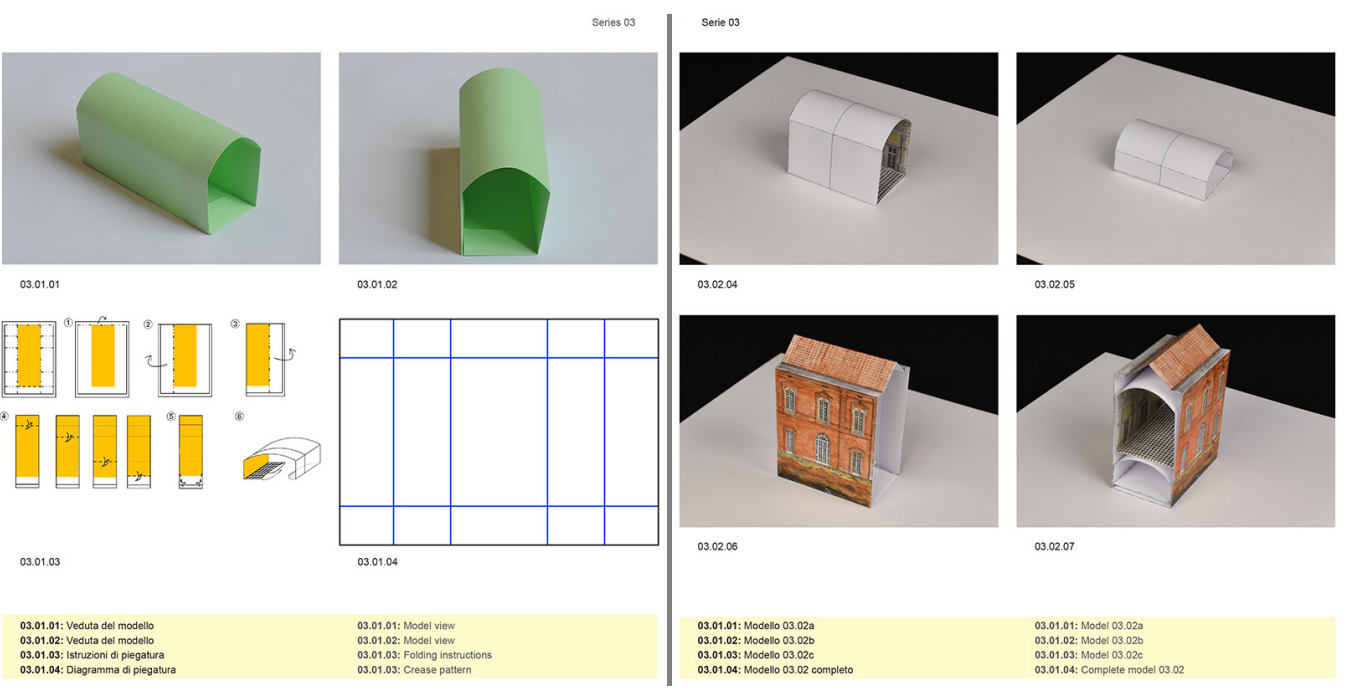



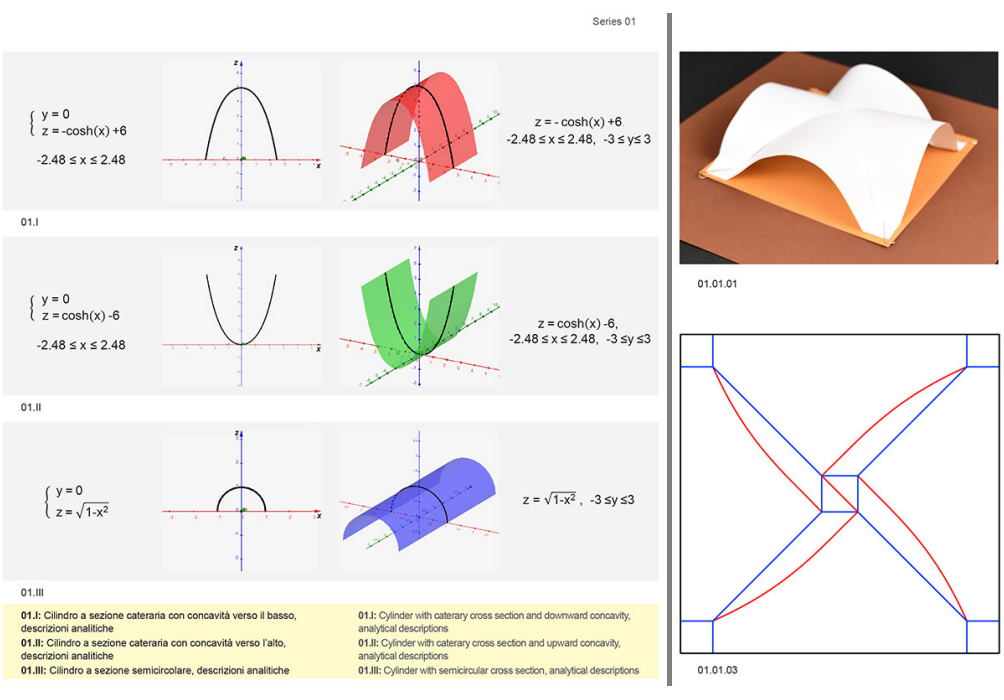

01.01 .01
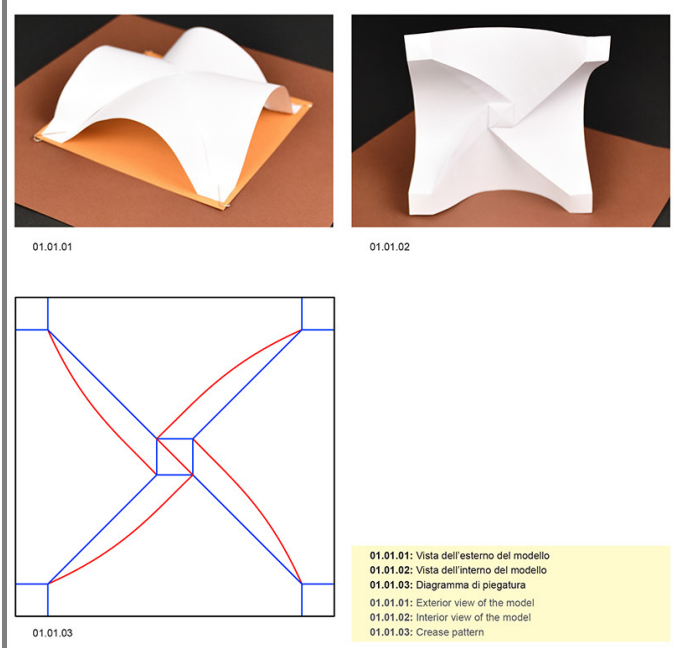

01.01 .02

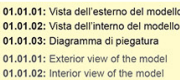

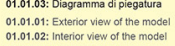

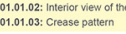

the laser cut one: this is achieved through the generalization of the constructive geometric process which is released from the real shape to become a tool for visualizing a theoretical shape (fig. 3). In the revision of the produced materials, more space was given to models of architectural shapes: in addition to the 00 series, referring to already known origami models, eleven of the twelve series are specific. The cataloged models are iconic artifacts, suitable for displaying architectures or parts of them, here reduced to a minimum and deprived of every element other than its own volume, on some occasions even left unfinished and defined only by intersections of surfaces. In a crescendo of difficulty, all models offer the possibility of giving shape to the volume through a few operations and can therefore be placed among the possible teaching aids for different ages of development, from the preoperative stage of Piaget (1955) to the university level. The 03 series presents a set of artifacts suitable for educating the mind also in the discretization of the data to be represented: through physical modeling, the user correlates shape and representation, overcoming the limits of its interpretation. The folding sequence makes use of a coded graphic language supported by intuitive representations so as to make its use feasible both in experiential teaching activities and in the training of trainers [Spreafico, Zich 2017]. The models, partly static and partly dynamic, are conceived as reproducible and dismountable objects. The origami approach allows the creation of both symbolic models and models that respect the geometric rigor of surfaces. For example, model 03.01 represents a barrel vault: it can be iconic, without geometric values, capable of changing section as the amount of paper inserted in the base pocket varies, but also rigorously constructed, to illustrate a specific geometry despite paper flexibility. Origami modeling makes the model suitable for experiential teaching at different levels and also useful for introducing the first notions of architectural language: here, the intersection between the cylindrical surface and the vertical wall represents the springer
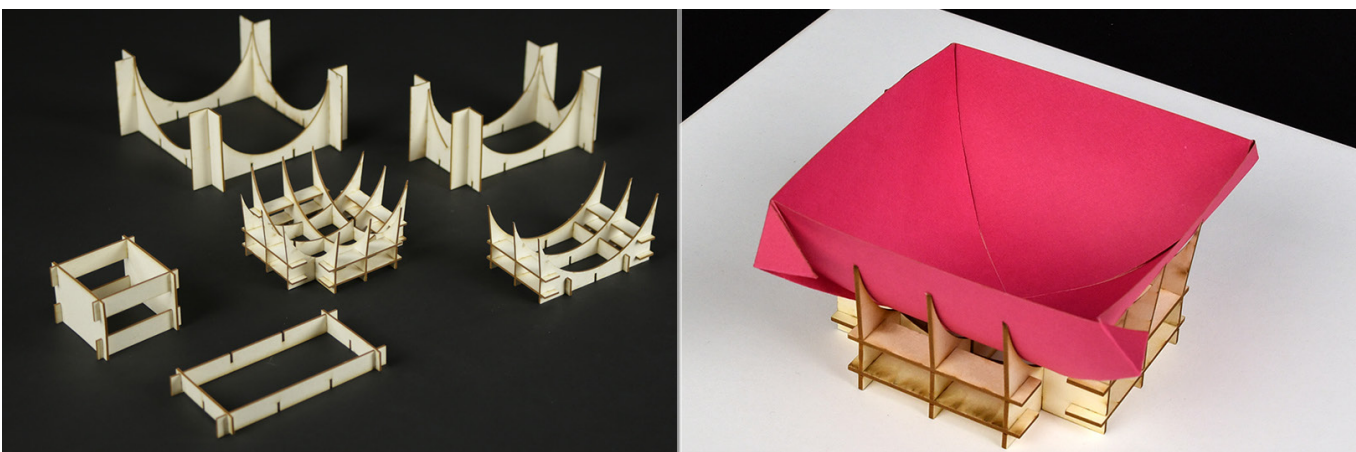
Fig. 7. Example of evolution of a groin vault model: 01.01 , catenary section-origami; 03.07, semicircular section-origami; semicircular section-laser

Fig. 8. Hyperbolic paraboloid, files: 10.01 , horizonta cutting planes-laser cut 10.03,3D print; visual and tangible interaction between different typology models of the same object can enhance user's comprehension and perception of the theoretical shapes.

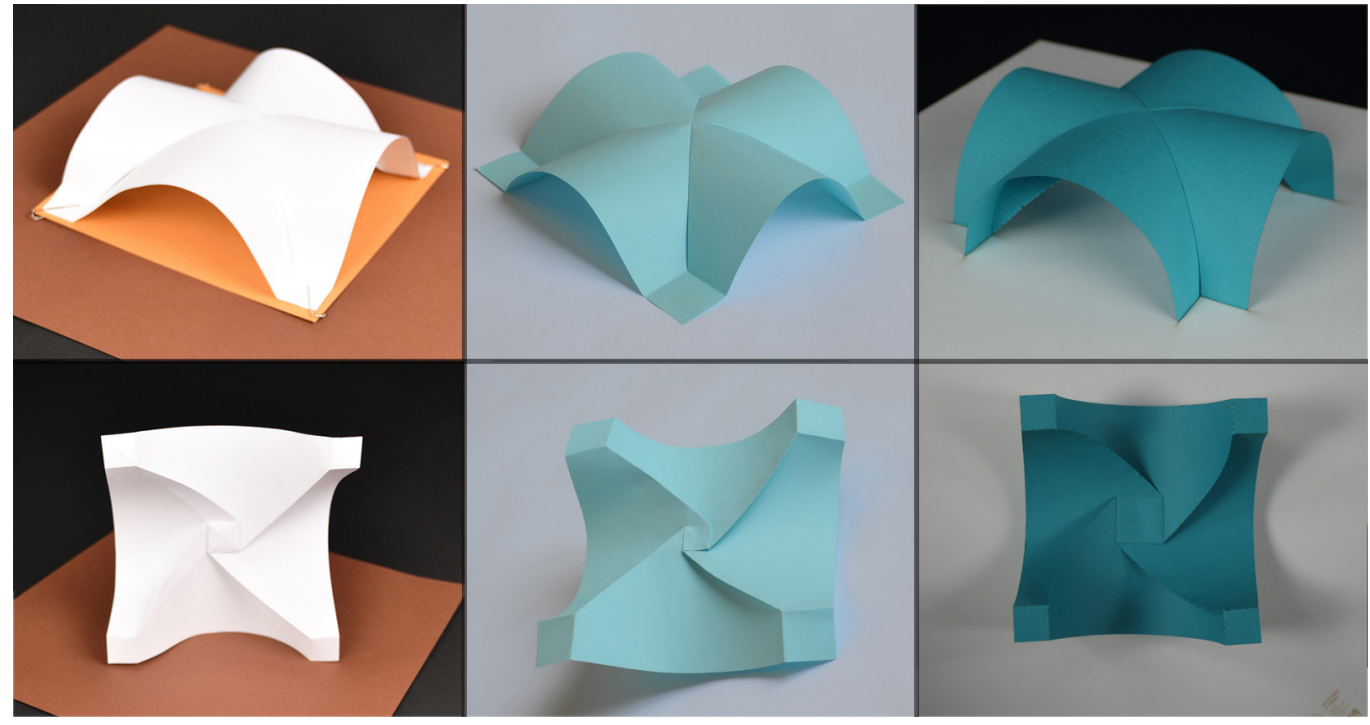

Serie 10 10.01 Paraboloide iperbolico (sella), piani sezione orizzontal
10.01 Hyperbolic paraboloid, horizontal cutting planes Idea | Concept
Progetto | Design
Modello | Model Tecnical Technique
Materiale | Material Dimensioni CP |
Fonti | Sources MP cartvegegale $2 \mathrm{~mm} \mid$ finn board $2 \mathrm{~mm}+\mathrm{MDF} 4 \mathrm{~mm}$ cartvegealale
varie |varies

I modello rappresenta una porzione della supericie
di equazione $z=x^{2} / \mathrm{a}^{2}-\mathrm{y}^{2} / \mathrm{b}^{2}$ attraverso una scelta $\begin{gathered}\text { The model represents a portion of the surface } \\ \text { descibed by the equation } z=x^{2} / \mathrm{a}^{2} \cdot \mathrm{y}^{2} / \mathrm{b}^{2} \text { via a selection }\end{gathered}$ piano $z=0, e$ una coppia di rete, mentre ei intersezioni
con i piani $z=k \neq 0$ sono iperboli le cui proiezioni sul
lines; on the other hand, intersections with horizontal

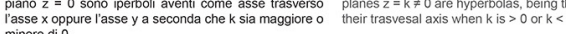
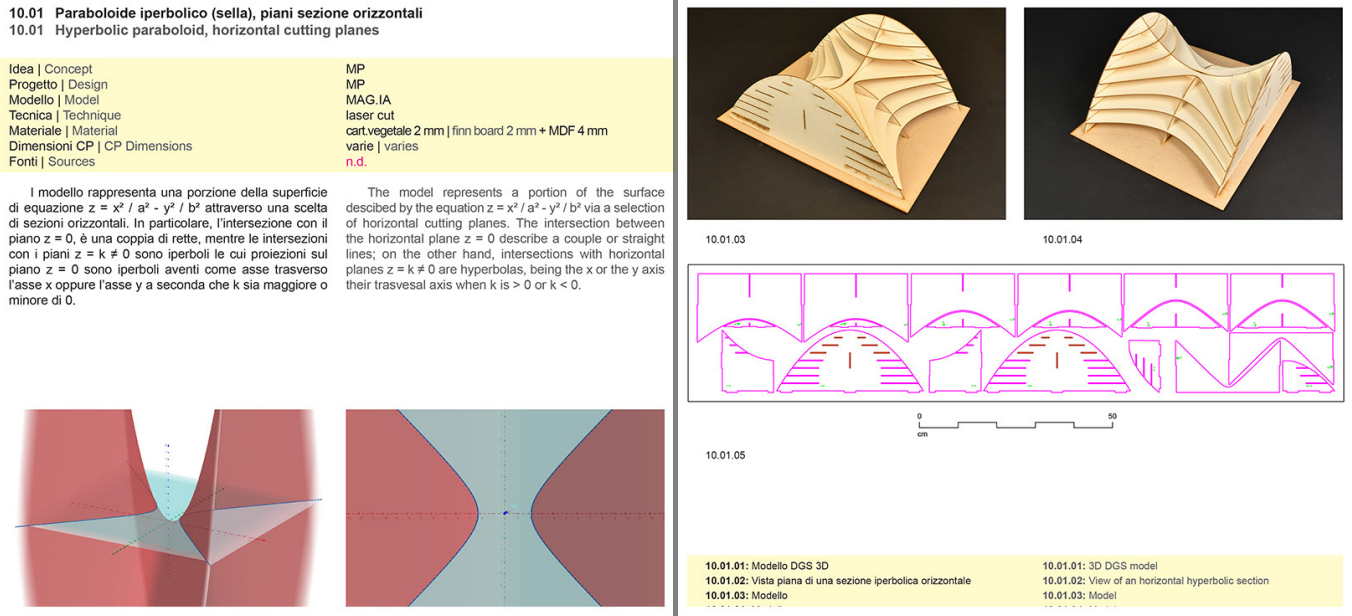

Series 10

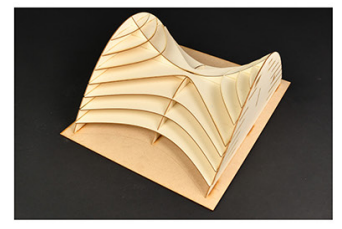


plane and highlights a straight line indicating the translation direction of the arc/directrix curve; the ground line is defined by the intersection between the vertical wall and the floor; the inner surface is the intrados, the outer surface is the extrados (fig. 4). The 01 series presents different types of vaults generated by the intersection of two cylinders; their spatial configuration is the result of an initial search for constraints in order to rigorously control the geometric process of development of the ruled surfaces (fig. 5). The 03 series revisits them by varying their basic geometries and the 12 series proposes them again by changing their production technique, with laser cut printing process, creation of supports for 'shaping', processing of the material support in order to facilitate the fold without weakening its structure (fig. 6) by varying accessibility/reproducibility and geometric rigor.To better manage the theoretical geometries, in the $\mathrm{OI}$ series there are more solutions of the same type of vault and each model has its own communicative specificities made explicit by the management of excess paper to underline (or partially hide) potential/limits of the plastic representation, while in series 12 the optimization process has reduced the number of possible solutions to the best ones for the chosen tool (fig. 7). With the aim of evaluating the communicative effectiveness of different production methods of the same model, the series 10 compares models obtained by 3D printer or as a sequence of plane sections with the laser cutter, that is used therefore no longer to manage developed surfaces, but to define a skeleton on which one can read the skin of the shape to be described (fig. 8). The series 09 summarizes the process of translating a built shape into a physical model to convey its geometric peculiarities to a non-specialist user, underlining the debate around how many and which models are useful to describe which geometry for which user, to create and visualize the more plausible theoretical surface to describe its complexity: the model of the built, with all its irregularities, would not help to grasp the geometric specificities of the surface (fig. 9). There are many models that address the relationship between the built architecture and the physical model in its iconic meaning and therefore do not have to manage these irregularities; in others, the built architecture is instead investigated through the critical reading of graphic sources and the successful recognition of the theoretical surfaces more similar to it.

Fig. 9. From the real shape to the tangible model. Benedetto Alfier's conoidical vault, Royal Palace of Venaria Reale (TO).
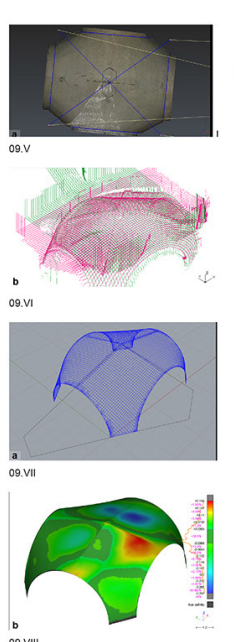

(ov)

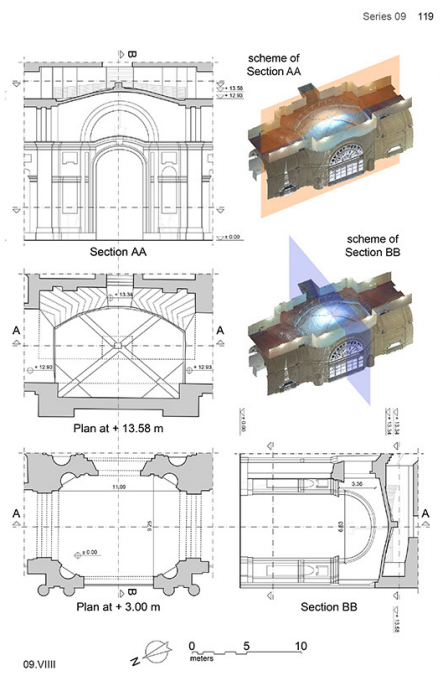

新

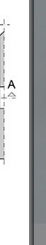

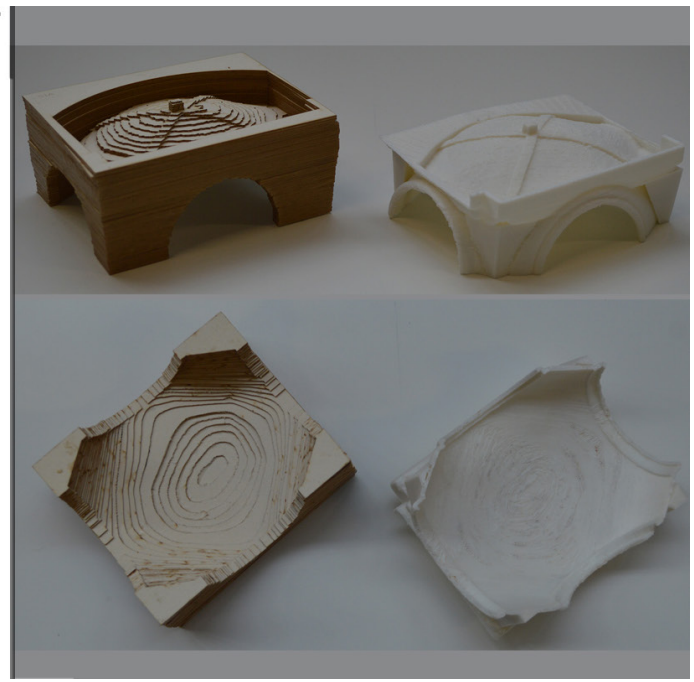

\section{Conclusions}

The critical re-reading of some catalogs of mathematical surfaces has inspired the drawing up of a visual catalog of models following a design process that adapts contents according to the production process and its aptitude for sharing concepts, taking into account the different intelligences involved. The models are obviously symbolic, they are representations without thickness of the theoretical surfaces that make up architectural shapes structure, 
presented with a descriptive and design graphic apparatus that makes them shareable. Production techniques range from classic origami based on a folding sequence to the use of laser cut that directly imprint all the folds on a sheet, from 3D printing to modeling with Dynamic Geometry Software (GeoGebra). The catalog underlines its communicative aims as well as the descriptive geometric ones and proposes the comparison between models of the same shape solved with different techniques and approaches to educate to architectural shape reading.

The relationship between the theoretical surface, physical model and built architecture, whether it is directly measured or interpreted through the critical reading of graphical sources, finds space in the catalog in compliance with the purposes of the same: the possibility of theoretically and operationally accompanying the production of models with different communicative registers.

In the increasingly current dynamics of distance learning, the experimentation of on-line laboratory activities has confirmed their effectiveness. All the models in the catalog are suitable tools to educate the mind to volumes perception and to discretize the data to be represented, therefore they can be proposed by teachers in different moments and educational contexts, at different levels.

\section{Acknowledgments}

We thank the ModLab Architecture (DAD - PoliTo) for supporting prototyping, the RilDoc (DAD - PoliTo) for supporting photographic reproduction, all the students who intervened from 2015 to today in the research projects that have led to the creation of the presented models.

C. Cumino is a member of the National Group for Algebraic and Geometric Structures and their Applications (GNSAGA-INDAM). For M. Pavignano and U. Zich the contribution was developed within the research project MAG.IA 2020: Mathematics Architecture Geometry. Interconnections and Applications.

\section{Notes}

[I] <https://sbs.uniromal .it/sapienzadigitallibrary> (accessed 202I, February 20).

[2] <www.eco.unina.it> (accessed 2021, February 20).

[3] <www.phaidra.cab.unipd.it> (accessed 2021, February 20).

[4] <www.retemuseiuniversitari.unimore.it> (accessed 2021, February 20).

[5] <www.unimuseum.uni-tuebingen.de> (accessed 202I, February 20).

\section{References}

Cardone V. (20 I 6). Imagining the cultural area of the visual images. In XY digitale, I ( ), pp. I2-27.

Cumino C. et al. (2017). Modelli cartacei per la comprensione della forma architettonica: ricerca, progetto, sperimentazione e didattica in un dialogo tra geometria e rappresentazione. In A Di Luggo et al. (a cura di). Territori e frontiere della rappresentazione. Atti del $39^{\circ}$ Convegno Internazionale dei Docenti delle Discipline della Rappresentazione. Napoli, I 4 - I 6 settembre 2017, pp. I279- I286. Roma: Gangemi.

Cumino C. et al. (2020). Geometry to Build Models, Models to Visualize Geometry. In Digital Experiences in Mathematics Education, pp. $149-166$.

De Fusco R. (20 I0). Architecturminimum: Le basi dello storicismo, strutturalismo, semiotica, ermeneutia \& altre teorie. Napoli: Clean Edizioni.

DickW. ( 1892). Katalog mathematischer und mathematisch-physikalischer Modelle, Apparate und Instrumente. München: K. Hof. u. Universitäts Buchdruckerei von Dr. C. Wolf \& Sohn.

Duval R. (2006). A cognitive analysis of problems of comprehension in the learning of mathematics. In Educational Studies in Mathematics, 61 ( I-2), pp. I03-131.

Emch A. (1920). Mathematical models. In University of Illinois Bulletin, XVIII (I2).

Emch A. (1923). Mathematical models II series. In University of Illinois Bulletin, XX (42).

Emch A. (1925). Mathematical models III series. In University of Illinois Bulletin, XXII (35).

Emch A. (1928). Mathematical models IV series. In University of Illinois Bulletin, XXV (43).

Estanave E. (s.d.). Construction des modèles de surfaces applicables sur le paraboloide de révolution dèfinies par M. G. Darboux. Paris: Gauthier-Villars. 
Fabre de Lagrange M., Merrifield C.W. (1872). A catalogue of a collection of models of ruled surfaces. London: George E. Eyre and William Spottiswoode.

Fehr H. F. (1938). The construction and use of mathematical models. Upper Montclair: s.e.

Gay F. (20 I7). Immagini che parlano di immagini. In A. Luigini (a cura di). Lineis Describere. Sette seminari tra rappresentazione e formazione, pp. 94- I 17. Melfi: Libria.

Gay F. (2000). Intorno agli Omolografi. Strumenti e Modelli per la Geometria Descrittiva. Venezia: IUAV.

Giacardi L. (20I5). Geometric Models in Mathematics Teaching in Italy at the Turn of the Twentieth Century. In Mathematisches Forschungsinstitut Oberwolfach, 47, pp. 2784-2787.

Guarini G. (1737). Architettura civile. Torino: Gianfrancesco Mairesse.

Innocenti P. (2007). A proposito di Bucherkunde: I. La Bibliographia di Blum, 2. II Fra biblioteca e archivio di Cavallaro, 3. Due recensioni di Serrai: una risata le seppelli. In Culture del testo e del documento, n. 25, pp. 27-70.

Maier H. (1989). Conflit entre langue mathématique et langue quotidienne pour les élèves. In Cahiers de didactique des mathématiques, 3, pp. 86-1 18.

Neuendorff R. (1922). Praktische mathematik. Leipzig: B. G. Teubner.

Pavignano M., Cumino C., Zich U. (2020). Catalog Mathematischer Modelle: Connessioni tra testo, rappresentazione grafica e descrizione analitica. In A. Arena et al. (a cura di). Connettere. Un disegno per annodare e tessere. Atti del $42^{\circ}$ Convegno internazionale dei Docenti delle Discipline della Rappresentazione, pp. 3660-3677. Milano: Franco Angeli.

Pavignano M., Zich U. (2020). Colore tra forma e materia dei modelli fisici per lo studio della Geometria. In V. Marchiafava, M. Piccolo (a cura di). Colore e Colorimetria. Contributi Multidisciplinari, vol. XVI A, pp. 398-405. Milano: Gruppo del Colore Associazione Italiana Colore.

Quaroni L. (1978). Progettare un edificio: Otto lezioni di architettura. Milano: Mazzotta.

Schilling M. (1903). Catalog mathematischer Modelle für den höheren mathematischen Unterricht. Halle: Martin Schilling.

Schilling M. (191 I). Catalog mathematischer Modelle für den höheren mathematischen Unterricht. Leipzig: Martin Schilling.

Sdegno A. et al. (20 17). Modellare smorfie. Rilievo e rappresentazione aptica di due teste scultoree di Franz Xavier Messerschmidt. In A Di Luggo et al. (a cura di). Territori e frontiere della rappresentazione. Atti del $39^{\circ}$ Convegno Internazionale dei Docenti delle Discipline della Rappresentazione. Napoli, I4- 16 settembre 20 17, pp. 969-976. Roma: Gangemi.

Seidl E., Loose F., Bierende. E. (ed.) (20।8). Mathematik mit Modellen. Alexander von Brill und die Tübinger Modell Sammlung. Tübingen: Museum der Universität Tübingen.

Sfard A. (2008). Thinking as communicating: Human development, the growth of discourses, and mathematizing. Cambridge University Press.

Spreafico M. L., Zich U. (20 17). Train the trainers on learn geometry by doing. In: L. Gómez Chova, A. López Martínez, I. Candel Torres (a cura di). EDULEARN 1 7. Proceedings 9th International Conference on Education and New Learning Technologies. Barcelona 3-5 July 2017, pp. 1969-1976. Valencia: IATED.

Stoll R. (|96I). Lehrmodelle für Mathematik. Berlin: Rudolf Stoll K.G.

Tagliari A., Florio W. (2013). Digital fabrication of physical models to analyze unbuilt projects using laser cutter. In P. J. da Silva Bartolo et al. (a cura di). High Value Manufacturing: Advanced Research in Virtual and Rapid Prototyping. Proceedings of the 6th International Conference on Advanced Research in Virtual and Rapid Prototyping. Leiria, Portugal, I-5 October 20 I 3. Boca Raton (FL): CRC Press.

Wiener H. (1907). Abhandlungen zur Sammlung mathematischer Modelle. Leipzig: B. G. Teubner.

Wiener H. ( I9II). Abhandlungen zur Sammlung mathematischer Modelle. Leipzig: B. G. Teubner.

Wiener H., Treutlein, P. (1912). Verzeichnis mathematischer Modelle. Leipzig: B. G. Teubner.

\section{Authors}

Caterina Cumino, Politecnico di Torino, caterina.cumino@polito.it

Martino Pavignano, Politecnico di Torino, martino.pavignano@polito.it

Ursula Zich, Politecnico di Torino, ursula.zich@polito.it

To cite this chapter. Cumino Caterina, Pavignano Martino, Zich Ursula (202I). Proposta di un catalogo visuale di modelli per lo studio della forma architettonica tra Matematica e Disegno/Visual catalog of models for the study of architectural shapes between Mathematics and Drawing: a new proposal. In Arena A., Arena M., Mediati D., Raffa P. (a cura di). Connettere. Un disegno per annodare e tessere. Linguaggi Distanze Tecnologie. Atti del $42^{\circ}$ Convegno Internazionale dei Docenti delle Discipline della Rappresentazione/Connecting. Drawing for weaving relationship. Languages Distances Technologies. Proceedings of the $42^{\text {th }}$ International Conference of Representation Disciplines Teachers. Milano: FrancoAngeli, pp. 626-645. 